\title{
Quantitative Analysis of Minium and Vermilion Mixtures Using Low-Frequency Vibrational Spectroscopy
}

Elyse M. Kleist \& Timothy M. Korter*

Department of Chemistry, 1-014 Center for Science and Technology, Syracuse University, Syracuse, NY 13244-4100 USA

*tmkorter@syr.edu

\section{Supporting Information Contents}

\section{X-ray Powder Diffraction Methodology}

\section{Computational Details}

Figure S1. Powder X-ray diffraction data (red) of minium (top panel) and vermilion (bottom panel) samples compared to the predicted powder patterns (black) based on the single-crystal structural data. SR1, SR2

Table S1. Pellet thickness (in millimeters) for pure and mixed sample pellets. Each pellet weighed approximately $800 \mathrm{mgs}$.

Table S2. SS-DFT simulated dielectric tensor components for minium and vermilion.

Table S3. Comparison of experimental and calculated lattice parameters for minium and vermilion using the PBE and PBE0 density functionals.

Table S4. Solid-state DFT simulated IR-active frequencies (including LO/TO splitting) for minium.

Table S5. Solid-state DFT simulated Raman-active frequencies and normalized intensities (including LO/TO splitting) for minium.

Figure S2. THz-TDS spectra simulated with PBE were convolved using empirically determined Lorentzian line shapes with a FWHM of $2.15 \mathrm{~cm}^{-1}$ for minium (top-panel) and $1.85 \mathrm{~cm}^{-1}$ for vermilion (bottom panel).

Table S6. Solid-state DFT simulated IR-active frequencies (including LO/TO splitting) for vermilion.

Table S7. Solid-state DFT simulated Raman-active frequencies and normalized intensities (including LO/TO splitting) for vermilion.

Figure S3. LFRS spectra simulated with PBE were convolved using Voigt profiles for minium (top-panel) and vermilion (bottom panel).

Figure S4. Experimental $290 \mathrm{~K} \mathrm{THz-TDS} \mathrm{spectra} \mathrm{of} \mathrm{pure} \mathrm{vermilion} \mathrm{(top)} \mathrm{and} \mathrm{minium} \mathrm{(bottom).}$

Figure S5. Peak fitting with Lorentzian line shapes of specific THz-TDS spectral range in minium and vermilion mixture sample, $1: 2$, using Origin $2018 b^{\mathrm{SR} 3}$. 
Figure S6. Peak fitting with Lorentzian line shapes of specific THz-TDS spectral range in minium and vermilion mixture sample, 2:3, using Origin $2018 \mathrm{~b}^{\mathrm{SR} 3}$.

Figure S7. Peak fitting with Lorentzian line shapes of specific THz-TDS spectral range in minium and vermilion mixture sample, $1: 1$, using Origin $2018 \mathrm{~b}^{\mathrm{SR} 3}$.

Figure S8. Peak fitting with Lorentzian line shapes of specific THz-TDS spectral range in minium and vermilion mixture sample, $3: 2$, using Origin $2018 b^{\mathrm{SR} 3}$.

Figure S9. Peak fitting with Lorentzian line shapes of specific THz-TDS spectral range in minium and vermilion mixture sample, $2: 1$, using Origin $2018 \mathrm{~b}^{\mathrm{SR} 3}$.

Figure S10. Parameters for linear least squares fit of THz-TDS calibration curves for minium (top) and vermilion (bottom).

Figure S11. Experimental 290 K LFRS spectra of pure vermilion (top) and minium (bottom) before normalization.

Figure S12. Peak fitting with Voigt line shapes of specific LFRS spectral range in minium and vermilion mixture sample, $1: 2$, using Origin $2018 \mathrm{~b}^{\mathrm{SR} 3}$.

Figure S13. Peak fitting with Voigt line shapes of specific LFRS spectral range in minium and vermilion mixture sample, 2:3, using Origin $2018 b^{\text {SR3 }}$

Figure S14. Peak fitting with Voigt line shapes of specific LFRS spectral range in minium and vermilion mixture sample, $1: 1$, using Origin $2018 b^{\mathrm{SR} 3}$.

Figure S15. Peak fitting with Voigt line shapes of specific LFRS spectral range in minium and vermilion mixture sample, $3: 2$, using Origin $2018 b^{\text {SR3 }}$.

Figure S16. Peak fitting with Voigt line shapes of specific LFRS spectral range in minium and vermilion mixture sample, $2: 1$, using Origin $2018 b^{\mathrm{SR} 3}$.

Figure S17. Parameters for linear least squares fit of LFRS calibration curves for minium (top) and vermilion (bottom).

Table S8. Experimental ${ }^{\mathrm{SR} 2}$ and solid-state DFT calculated bonds lengths and angles for minium asymmetric unit.

Table S9. Experimental ${ }^{\mathrm{SR} 1}$ and solid-state DFT calculated bonds length for vermilion asymmetric unit.

Figure S18. Eigenvector plot for Raman-active mode, $22.43 \mathrm{~cm}^{-1}$, in minium.

Figure S19. Eigenvector plot for Raman-active mode, $57.37 \mathrm{~cm}^{-1}$, in minium.

Figure S20. Eigenvector plot for IR-active mode, $58.22 \mathrm{~cm}^{-1}$, in minium.

Figure S21. Eigenvector plot for IR-active mode, $60.23 \mathrm{~cm}^{-1}$, in minium.

Figure S22. Eigenvector plot for Raman-active mode, $66.95 \mathrm{~cm}^{-1}$, in minium.

Figure S23. Eigenvector plot for Raman-active mode, $72.11 \mathrm{~cm}^{-1}$, in minium.

Figure S24. Eigenvector plot for Raman-active mode, $72.91 \mathrm{~cm}^{-1}$, in minium.

Figure S25. Eigenvector plot for IR-active mode, $74.17 \mathrm{~cm}^{-1}$, in minium. 
Figure S26. Eigenvector plot for IR-active mode, $85.77 \mathrm{~cm}^{-1}$, in minium.

Figure S27. Eigenvector plot for Raman -active mode, $88.06 \mathrm{~cm}^{-1}$, in minium.

Figure S28. Eigenvector plot for Raman -active mode, $89.24 \mathrm{~cm}^{-1}$, in minium.

Figure S29. Eigenvector plot for IR-active mode, $116.19 \mathrm{~cm}^{-1}$, in minium.

Figure S30. Eigenvector plot for Raman-active mode, $121.67 \mathrm{~cm}^{-1}$, in minium.

Figure S31. Eigenvector plot for Raman-active mode, $128.60 \mathrm{~cm}^{-1}$, in minium.

Figure S32. Eigenvector plot for IR-active mode, $130.83 \mathrm{~cm}^{-1}$, in minium.

Figure S33. Eigenvector plot for Raman-active mode, $132.15 \mathrm{~cm}^{-1}$, in minium.

Figure S34. Eigenvector plot for Raman-active mode, $158.79 \mathrm{~cm}^{-1}$, in minium.

Figure S35. Eigenvector plot for Raman-active mode, $169.84 \mathrm{~cm}^{-1}$, in minium.

Figure S36. Eigenvector plot for IR-active mode, $35.08 \mathrm{~cm}^{-1}$, in vermilion.

Figure S37. Eigenvector plot for Raman-active mode, $45.86 \mathrm{~cm}^{-1}$, in vermilion.

Figure S38. Eigenvector plot for Raman \& IR-active mode, $46.52 \mathrm{~cm}^{-1}$, in vermilion.

Figure S39. Eigenvector plot for Raman \& IR-active mode, $90.61 \mathrm{~cm}^{-1}$, in vermilion.

Figure S40. Eigenvector plot for Raman \& IR-active mode, $111.27 \mathrm{~cm}^{-1}$, in vermilion.

Figure S41. Eigenvector plot for IR-active mode, $111.54 \mathrm{~cm}^{-1}$, in vermilion.

\section{Supporting Information References}

SR1. Auvray, P.; Genet, F., Refining the Crystal Structure of Cinnabar, $\alpha$-Mercury(II) Sulfide. Bull. Soc. Fr. Mineral. Cristallogr. 1973, 96 (3), 218-19.

SR2. Gavarri, J.-R.; Weigel, D., Oxydes de Plomb. I. Structure Cristalline du Minium $\mathrm{Pb}_{3} \mathrm{O}_{4}$, à Température Ambiante (293 K). J. Solid State Chem. 1975, 13 (3), 252-257.

SR3. OriginLab Corporation, OriginLab 2018b. (Northampton, MA).

SR4. Macrae, C. F.; Bruno, I. J.; Chisholm, J. A.; Edgington, P. R.; McCabe, P.; Pidcock, E.; Rodriguez-Monge, L.; Taylor, R.; van de Streek, J.; Wood, P. A., Mercury CSD 2.0 - New Features for the Visualization and Investigation of Crystal Structures. J. Appl. Crystallogr. 2008, 41 (2), 466-470.

SR5. Perdew, J. P.; Burke, K.; Ernzerhof, M., Generalized Gradient Approximation Made Simple. Phys. Rev. Lett. 1996, 77 (18), 3865-3868.

SR6. Adamo, C.; Barone, V., Toward Reliable Density Functional Methods Without Adjustable Parameters: The PBE0 Model. J. Chem. Phys. 1999, 110 (13), 6158-6170.

SR7. Peintinger, M. F.; Oliveira, D. V.; Bredow, T., Consistent Gaussian Basis Sets of TripleZeta Valence with Polarization Quality for Solid-State Calculations. J. Comput. Chem. 2013, 34 (6), 451-459.

SR8. Zagorac, D.; Doll, K.; Schön, J. C.; Jansen, M., Ab Initio Structure Prediction for Lead Sulfide at Standard and Elevated Pressures. Phys. Rev. B 2011, 84 (4), 045206. 
SR9. Metz, B.; Stoll, H.; Dolg, M., Small-Core Multiconfiguration-Dirac-Hartree-FockAdjusted Pseudopotentials for Post-d Main Group Elements: Application to $\mathrm{PbH}$ and $\mathrm{PbO} . J$. Chem. Phys. 2000, 113 (7), 2563-2569.

SR10. Weihrich, R.; Anusca, I.; Zabel, M., Halbantiperowskite: Zur Struktur der Shandite $\mathrm{M}_{3 / 2} \mathrm{AS}(\mathrm{M}=\mathrm{Co}, \mathrm{Ni} ; \mathrm{A}=\mathrm{In}, \mathrm{Sn})$ und ihren Typ-Antitypbeziehungen. Z. Anorg. Allgemeine Chem. 2005, 631 (8), 1463-1470.

SR11. Pascale, F.; Zicovich-Wilson, C. M.; Gejo, F. L.; Civalleri, B.; Orlando, R.; Dovesi, R., The Calculation of the Vibrational Frequencies of Crystalline Compounds and its Implementation in the CRYSTAL Code. J. Comput. Chem. 2004, 25 (6), 888-897. SR12. Zicovich-Wilson, C. M.; Pascale, F.; Roetti, C.; Saunders, V. R.; Orlando, R.; Dovesi, R., Calculation of the Vibration Frequencies of $\alpha$-Quartz: The Effect of Hamiltonian and Basis Set. J. Comp. Chem. 2004, 25 (15), 1873-1881.

SR13. Noel, Y.; Zicovich-Wilson, C. M.; Civalleri, B.; D'Arco, P.; Dovesi, R., Polarization Properties of $\mathrm{ZnO}$ and BeO: An Ab Initio Study Through the Berry Phase and Wannier Functions Approaches. Phys. Rev. B: Condens. Matter Mater. Phys. 2002, 65 (1), 1-9.

SR14. Maschio, L.; Kirtman, B.; Orlando, R.; Rerat, M., Ab Initio Analytical Infrared Intensities for Periodic Systems Through a Coupled Perturbed Hartree-Fock/Kohn-Sham Method. J. Chem. Phys. 2012, 137 (20), 204113/1-204113/11.

SR15. Maschio, L.; Kirtman, B.; Rérat, M.; Orlando, R.; Dovesi, R., Ab Initio Analytical Raman Intensities for Periodic Systems Through a Coupled Perturbed Hartree-Fock/Kohn-Sham Method in an Atomic Orbital Basis. II. Validation and Comparison with Experiments. J. Chem. Phys. 2013, 139 (16), 164102.

SR16. Ferrero, M.; Rérat, M.; Orlando, R.; Dovesi, R., The Calculation of Static Polarizabilities of 1-3D Periodic Compounds. The Implementation in the CRYSTAL Code. $J$. Comput. Chem. 2008, 29 (9), 1450-1459.

SR17. Ferrero, M.; Rérat, M.; Orlando, R.; Dovesi, R., Coupled Perturbed Hartree-Fock for Periodic Systems: The Role of Symmetry and Related Computational Aspects. J. Chem. Phys. 2008, $128(1), 014110$.

SR18. Ferrero, M.; Rérat, M.; Kirtman, B.; Dovesi, R., Calculation of First and Second Static Hyperpolarizabilities of One- to Three-Dimensional Periodic Compounds. Implementation in the CRYSTAL Code. J. Chem. Phys. 2008, 129 (24), 244110.

SR19. Monkhorst, H. J.; Pack, J. D., Special Points for Brillouin-Zone Integrations. Phys. Rev. B: Condens. Matter 1976, 13 (12), 5188-5192. 


\section{X-RAY POWDER DIFFRACTION METHODOLOGY}

The purity and bulk crystallinity of powdered minium and vermilion were verified by powder X-ray diffraction (PXRD) on a Bruker D2 Phaser with a LYKXEYE 1D silicon strip detector using $\mathrm{Cu}$ K $\alpha$ radiation $(\lambda=1.5406 \AA)$. Experimental PXRD patterns were compared to predicted patterns produced in Mercury ${ }^{\mathrm{SR} 4}$ using the published $290 \mathrm{~K}$ single-crystal X-ray diffraction data for minium $^{\mathrm{SR} 2}$ and vermilion. ${ }^{\mathrm{SR} 1}$

\section{COMPUTATIONAL DETAILS}

The $\mathrm{PBE}^{\mathrm{SR} 5}$ (Perdew-Burke-Ernzerhof) and $\mathrm{PBE0}^{\mathrm{SR} 6}$ density functionals were used with the POB-TZVP basis set ${ }^{\mathrm{SR} 7}$ for non-metal atoms (oxygen and sulfur) in both minium and vermilion, and effective core potential basis sets were used for lead ${ }^{\mathrm{SR} 8, \mathrm{SR} 9}$ and mercury ${ }^{\mathrm{SR} 10}$ atoms. Simulations for minium accounted for the coexisting divalent $\mathrm{Pb}$ (II) and tetravalent $\mathrm{Pb}$ (IV) atoms in the crystal lattice. Full geometry optimizations with an energy convergence of $\Delta \mathrm{E}<10^{-8}$ hartree were carried out using initial atomic positions, lattice dimensions, and space groups from the published roomtemperature crystallographic data. SR1, SR2 Harmonic vibrational analyses were performed on the optimized structures at the same level of theory, but with an energy convergence of $\Delta \mathrm{E}<10^{-10}$ hartree. Numerical derivatives for frequencies were calculated using the central-difference formula with two displacements per Cartesian axis, per atom. ${ }^{\text {SR11 }}$ Infrared intensities for generation of THz-TDS spectra were calculated using the CPHF analytical approach. ${ }^{\text {SR12, SR13, SR14 }}$ The infrared intensities $(\mathrm{km} / \mathrm{mol})$ were converted to extinction coefficients $\left(\mathrm{M}^{-1} \mathrm{~cm}^{-1}\right)$ for direct comparison to the experimental THz-TDS data. It was found that the simulations consistently overestimated the peak intensities and consequently the simulated THz-TDS intensities in this work have been scaled down by a factor of three for minium and five for vermilion to match observations. Simulated LFRS data utilized Raman tensors calculated with a coupled-perturbed Hartree-Fock/ Kohn-Sham 
approach and included effects from experimental conditions including temperature and incoming laser frequency. ${ }^{\text {SR14, SR15 }}$ The Raman scattering intensities have been normalized to 1000.0 based on the most intense feature in the entire calculated spectrum (found at $128.61 \mathrm{~cm}^{-1}$ for minium and $262.05 \mathrm{~cm}^{-1}$ for vermilion). Longitudinal optical-transverse optical (LO-TO) phonon splitting was evaluated in the frequency analyses through charge localization via Wannier functions and coupled-perturbed Hartree-Fock/ Kohn-Sham calculation of the high-frequency dielectric

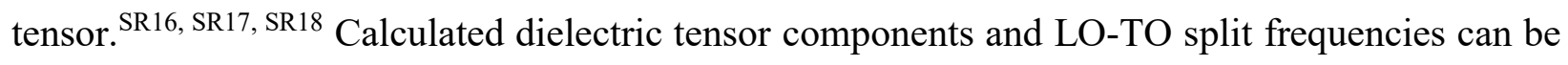
found inTable S3. The overlap-based truncation tolerances for the Coulomb and exchange series integrals were set to $10^{-12}, 10^{-12}, 10^{-12}, 10^{-15}$, and $10^{-30}$ hartree. The simulations used a pruned DFT integration grid comprised of 99 radial and 1454 angular points. The appropriate number of kpoints in the Monkhorst-Pack scheme, or the shrinking factor, ${ }^{\mathrm{SR} 19}$ was determined for each structure by monitoring total energy convergence for each solid as a function of k-point count. 

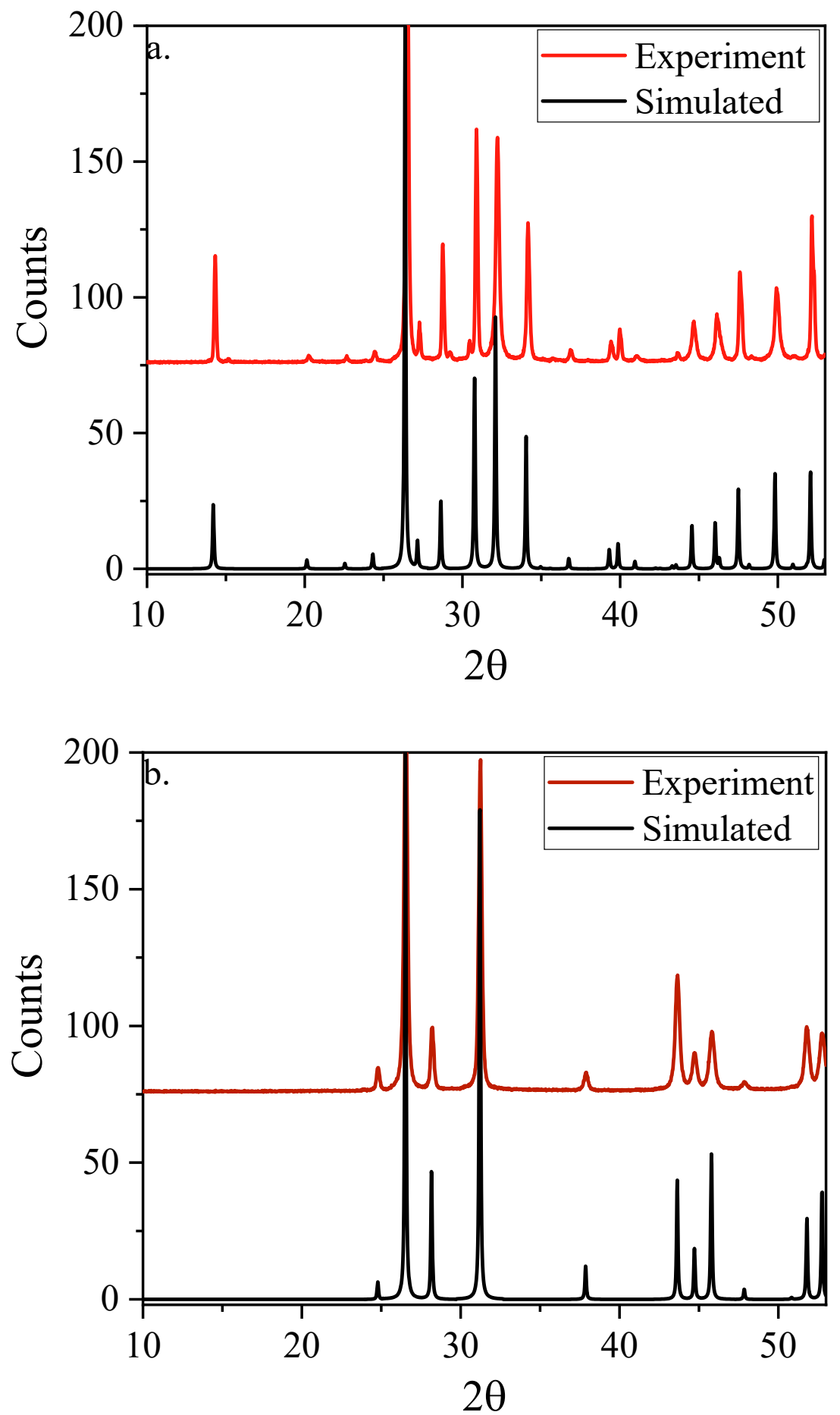

Figure S1. Powder X-ray diffraction data (red) of minium (a) and vermilion (b) samples compared to the predicted powder patterns (black) based on the single-crystal structural data. $\mathrm{SR} 1, \mathrm{SR} 2$ 
Table S1. Pellet thickness (in millimeters) for pure and mixed sample pellets. Each pellet weighed approximately $800 \mathrm{mgs}$.

\begin{tabular}{cc}
\hline & Pellet thickness $(\mathrm{mm})$ \\
\hline Minium & 2.89 \\
Vermilion & 2.82 \\
Blank (PTFE) & 2.90 \\
$3: 2$ & 2.85 \\
$1: 1$ & 2.86 \\
$2: 3$ & 2.95 \\
$1: 2$ & 2.56 \\
$2: 1$ & 2.70 \\
\hline
\end{tabular}

Table S2. SS-DFT simulated dielectric tensor components for minium and vermilion.

\begin{tabular}{|c|c|c|c|c|c|c|}
\hline & \multicolumn{3}{|c|}{ Minium } & \multicolumn{3}{|c|}{ Vermilion } \\
\hline & $\mathrm{XX}$ & YY & $\mathrm{ZZ}$ & $\mathrm{XX}$ & YY & $\mathrm{ZZ}$ \\
\hline PBE & 5.594 & 5.594 & 5.594 & 7.911 & 7.911 & 9.949 \\
\hline PBE0 & 4.620 & 4.620 & 4.726 & 6.249 & 6.249 & 7.494 \\
\hline
\end{tabular}


Table S3. Comparison of experimental and calculated lattice parameters for minium and vermilion using the PBE and PBE0 density functionals.

\begin{tabular}{l|lll|lll} 
& \multicolumn{3}{|l}{ Minium } & & \multicolumn{2}{l}{ Vermilion } \\
& Exp. $^{a}$ & PBE & PBE0 & Exp. & PBE & PBE0 \\
\hline \hline $\mathrm{a}(\AA)$ & 8.80 & 8.845 & 8.787 & 4.17 & 4.198 & 4.175 \\
$\mathrm{~b}(\AA)$ & 8.80 & 8.845 & 8.787 & 4.17 & 4.198 & 4.175 \\
$\mathrm{c}(\AA)$ & 6.62 & 6.778 & 6.622 & 9.61 & 9.684 & 9.597 \\
$\alpha\left(^{\circ}\right)$ & 90 & 90 & 90 & 90 & 90 & 90 \\
$\beta\left(^{\circ}\right)$ & 90 & 90 & 90 & 90 & 90 & 90 \\
$\gamma\left({ }^{\circ}\right)$ & 90 & 90 & 90 & 90 & 90 & 90 \\
$\mathrm{~V}\left(\AA^{3}\right)$ & 512.12 & 530.303 & 511.296 & 144.71 & 147.774 & 144.884 \\
\hline
\end{tabular}

\footnotetext{
${ }^{a}$ ref. SR1

${ }^{b}$ ref. SR2
} 
Table S4. Solid-state DFT simulated IR-active frequencies (including LO/TO splitting) for minium.

\begin{tabular}{|c|c|c|c|c|c|c|c|c|}
\hline & \multicolumn{4}{|c|}{ PBE } & \multicolumn{4}{|c|}{ PBE0 } \\
\hline & Fre & & Mode & Intensity & Freq & ency & Mode & Intensity \\
\hline & $(\mathrm{THz})$ & $\left(\mathrm{cm}^{-1}\right)$ & Symmetry & $(\mathrm{km} / \mathrm{mol})$ & $(\mathrm{THz})$ & $\left(\mathrm{cm}^{-1}\right)$ & Symmetry & $(\mathrm{km} / \mathrm{mol})$ \\
\hline \multirow{16}{*}{$\begin{array}{c}\text { Transverse } \\
\text { Optical } \\
\text { Modes }\end{array}$} & 1.61 & 53.55 & $\overline{E_{u}}$ & 92.18 & 1.75 & 58.22 & $\overline{E_{u}}$ & 35.72 \\
\hline & 1.73 & 57.56 & $\mathrm{E}_{\mathrm{u}}$ & 151.38 & 1.81 & 60.23 & $\mathrm{E}_{\mathrm{u}}$ & 220.35 \\
\hline & 1.99 & 66.25 & $\mathrm{~A}_{2 \mathrm{u}}$ & 79.91 & 2.22 & 74.17 & $\mathrm{~A}_{2 \mathrm{u}}$ & 40.81 \\
\hline & 2.33 & 77.85 & $\mathrm{E}_{\mathrm{u}}$ & 111.37 & 2.57 & 85.77 & $\mathrm{E}_{\mathrm{u}}$ & 100.03 \\
\hline & 3.18 & 105.94 & $\mathrm{~A}_{2 \mathrm{u}}$ & 365.30 & 3.48 & 116.19 & $\mathrm{E}_{\mathrm{u}}$ & 1.62 \\
\hline & 3.30 & 109.96 & $\mathrm{E}_{\mathrm{u}}$ & 0.31 & 3.92 & 130.83 & $\mathrm{~A}_{2 \mathrm{u}}$ & 449.88 \\
\hline & 4.30 & 143.53 & $\mathrm{E}_{\mathrm{u}}$ & 739.04 & 4.59 & 153.12 & $\mathrm{E}_{\mathrm{u}}$ & 829.10 \\
\hline & 5.67 & 189.26 & $\mathrm{E}_{\mathrm{u}}$ & 3.16 & 6.50 & 216.82 & $\mathrm{E}_{\mathrm{u}}$ & 43.09 \\
\hline & 8.04 & 268.21 & $\mathrm{E}_{\mathrm{u}}$ & 2584.91 & 8.51 & 283.78 & $\mathrm{E}_{\mathrm{u}}$ & 2796.69 \\
\hline & 9.31 & 310.52 & $\mathrm{E}_{\mathrm{u}}$ & 2693.80 & 9.82 & 327.68 & $\mathrm{E}_{\mathrm{u}}$ & 2450.93 \\
\hline & 9.94 & 331.49 & $\mathrm{~A}_{2 \mathrm{u}}$ & 5428.33 & 11.14 & 371.68 & $\mathrm{~A}_{2 \mathrm{u}}$ & 5905.17 \\
\hline & 11.10 & 370.24 & $\mathrm{E}_{\mathrm{u}}$ & 1235.97 & 11.88 & 396.14 & $\mathrm{E}_{\mathrm{u}}$ & 801.66 \\
\hline & 11.90 & 396.86 & $\mathrm{E}_{\mathrm{u}}$ & 3469.52 & 12.94 & 431.72 & $\mathrm{E}_{\mathrm{u}}$ & 4542.89 \\
\hline & 13.40 & 446.87 & $\mathrm{~A}_{2 \mathrm{u}}$ & 2237.98 & 14.17 & 472.59 & $\mathrm{~A}_{2 \mathrm{u}}$ & 1903.39 \\
\hline & 14.87 & 495.85 & $\mathrm{E}_{\mathrm{u}}$ & 802.10 & 16.13 & 538.06 & $\mathrm{E}_{\mathrm{u}}$ & 1640.97 \\
\hline & 15.25 & 508.75 & $\mathrm{E}_{\mathrm{u}}$ & 678.40 & 16.31 & 543.93 & $\mathrm{E}_{\mathrm{u}}$ & 42.67 \\
\hline \multirow{16}{*}{$\begin{array}{l}\text { Longitudinal } \\
\text { Optical } \\
\text { Modes }\end{array}$} & 1.64 & 54.66 & $\overline{E_{u}}$ & 1.80 & 1.75 & 58.45 & $\mathrm{E}_{\mathrm{u}}$ & 0.20 \\
\hline & 1.89 & 62.90 & $\mathrm{E}_{\mathrm{u}}$ & 29.20 & 2.02 & 67.42 & $\mathrm{E}_{\mathrm{u}}$ & 28.80 \\
\hline & 2.08 & 69.53 & $\mathrm{~A}_{2 \mathrm{u}}$ & 8.40 & 2.28 & 76.09 & $\mathrm{~A}_{2 \mathrm{u}}$ & 4.70 \\
\hline & 2.43 & 81.22 & $\mathrm{E}_{\mathrm{u}}$ & 33.40 & 2.66 & 88.81 & $\mathrm{E}_{\mathrm{u}}$ & 23.40 \\
\hline & 3.58 & 119.49 & $\mathrm{~A}_{2 \mathrm{u}}$ & 85.10 & 3.48 & 116.21 & $\mathrm{E}_{\mathrm{u}}$ & 0.20 \\
\hline & 3.30 & 109.97 & $\mathrm{E}_{\mathrm{u}}$ & 0.10 & 4.38 & 146.25 & $\mathrm{~A}_{2 \mathrm{u}}$ & 90.60 \\
\hline & 4.62 & 154.15 & $\mathrm{E}_{\mathrm{u}}$ & 179.80 & 4.97 & 165.82 & $\mathrm{E}_{\mathrm{u}}$ & 165.40 \\
\hline & 5.68 & 189.30 & $\mathrm{E}_{\mathrm{u}}$ & 0.90 & 6.51 & 217.27 & $\mathrm{E}_{\mathrm{u}}$ & 7.60 \\
\hline & 8.46 & 282.21 & $\mathrm{E}_{\mathrm{u}}$ & 254.10 & 9.01 & 300.45 & $\mathrm{E}_{\mathrm{u}}$ & 225.60 \\
\hline & 10.02 & 334.23 & $\mathrm{E}_{\mathrm{u}}$ & 816.10 & 10.64 & 354.91 & $\mathrm{E}_{\mathrm{u}}$ & 818.90 \\
\hline & 11.95 & 398.66 & $\mathrm{~A}_{2 \mathrm{u}}$ & 953.20 & 12.03 & 401.25 & $\mathrm{E}_{\mathrm{u}}$ & 154.60 \\
\hline & 11.30 & 376.85 & $\mathrm{E}_{\mathrm{u}}$ & 205.50 & 13.17 & 439.26 & $\mathrm{~A}_{2 \mathrm{u}}$ & 566.90 \\
\hline & 13.48 & 449.63 & $\mathrm{E}_{\mathrm{u}}$ & 4475.60 & 14.79 & 493.20 & $\mathrm{E}_{\mathrm{u}}$ & 3425.60 \\
\hline & 15.05 & 501.93 & $\mathrm{E}_{\mathrm{u}}$ & 376.10 & 16.30 & 543.78 & $\mathrm{E}_{\mathrm{u}}$ & 5.80 \\
\hline & 15.94 & 531.56 & $\mathrm{E}_{\mathrm{u}}$ & 6189.50 & 17.41 & 580.79 & $\mathrm{~A}_{2 \mathrm{u}}$ & 7637.00 \\
\hline & 16.01 & 534.01 & $\mathrm{~A}_{2 \mathrm{u}}$ & 7064.80 & 17.45 & 582.20 & $\mathrm{E}_{\mathrm{u}}$ & 8649.50 \\
\hline
\end{tabular}


Table S5. Solid-state DFT simulated Raman-active frequencies and normalized intensities (including LO/TO splitting) for minium.

\begin{tabular}{|c|c|c|c|c|c|c|c|c|}
\hline & \multicolumn{4}{|c|}{ PBE } & \multicolumn{4}{|c|}{ PBE0 } \\
\hline & \multirow{2}{*}{\multicolumn{2}{|c|}{$\begin{array}{l}\text { Frequency } \\
(\mathrm{THz}) \quad\left(\mathrm{cm}^{-1}\right)\end{array}$}} & \multirow{2}{*}{$\begin{array}{c}\text { Mode } \\
\text { Symmetry }\end{array}$} & \multirow{2}{*}{$\begin{array}{l}\text { Intensity } \\
(\mathrm{km} / \mathrm{mol})\end{array}$} & \multicolumn{2}{|c|}{ Frequency } & \multirow{2}{*}{$\begin{array}{c}\text { Mode } \\
\text { Symmetry }\end{array}$} & \multirow{2}{*}{$\begin{array}{l}\text { Intensity } \\
(\mathrm{km} / \mathrm{mol})\end{array}$} \\
\hline & & & & & $(\mathrm{THz})$ & $\left(\mathrm{cm}^{-1}\right)$ & & \\
\hline \multirow{26}{*}{$\begin{array}{l}\text { Transverse } \\
\text { Optical } \\
\text { Modes }\end{array}$} & 1.61 & 53.85 & $\mathrm{~B}_{1 \mathrm{~g}}$ & 87.78 & 0.67 & 22.43 & $E_{g}$ & 6.59 \\
\hline & 1.84 & 61.47 & $\mathrm{E}_{\mathrm{g}}$ & 74.75 & 1.72 & 57.37 & $\mathrm{~B}_{1 \mathrm{~g}}$ & 52.86 \\
\hline & 1.97 & 65.76 & $\mathrm{~A}_{1 \mathrm{~g}}$ & 6.29 & 2.01 & 66.95 & $E_{g}$ & 120.32 \\
\hline & 2.00 & 66.79 & $\mathrm{~B}_{1 \mathrm{~g}}$ & 1.06 & 2.16 & 72.11 & $\mathrm{~B}_{1 \mathrm{~g}}$ & 0.41 \\
\hline & 2.27 & 75.88 & $\mathrm{E}_{\mathrm{g}}$ & 0.14 & 2.19 & 72.91 & $\mathrm{~A}_{1 \mathrm{~g}}$ & 19.64 \\
\hline & 2.54 & 84.77 & $\mathrm{~B}_{2 \mathrm{~g}}$ & 34.46 & 2.64 & 88.06 & $\mathrm{~B}_{2 \mathrm{~g}}$ & 14.62 \\
\hline & 3.10 & 103.48 & $\mathrm{E}_{\mathrm{g}}$ & 10.65 & 2.68 & 89.24 & $\mathrm{E}_{\mathrm{g}}$ & 8.74 \\
\hline & 3.61 & 120.49 & $\mathrm{~A}_{1 \mathrm{~g}}$ & 1000.00 & 3.65 & 121.67 & $E_{g}$ & 10.80 \\
\hline & 3.69 & 123.25 & $B_{2 g}$ & 5.01 & 3.86 & 128.61 & $\mathrm{~A}_{1 \mathrm{~g}}$ & 1000.00 \\
\hline & 4.45 & 148.30 & $\mathrm{E}_{\mathrm{g}}$ & 29.53 & 3.96 & 132.15 & $\mathrm{~B}_{2 \mathrm{~g}}$ & 20.53 \\
\hline & 4.55 & 151.61 & $\mathrm{~B}_{1 \mathrm{~g}}$ & 0.08 & 4.76 & 158.79 & $\mathrm{E}_{\mathrm{g}}$ & 63.40 \\
\hline & 6.19 & 206.34 & $\mathrm{~B}_{1 \mathrm{~g}}$ & 18.44 & 5.09 & 169.84 & $\mathrm{~B}_{1 \mathrm{~g}}$ & 8.50 \\
\hline & 6.93 & 231.08 & $A_{1 g}$ & 36.96 & 7.02 & 234.02 & $\mathrm{~B}_{1 \mathrm{~g}}$ & 14.17 \\
\hline & 7.45 & 248.56 & $\mathrm{~B}_{1 \mathrm{~g}}$ & 0.10 & 7.11 & 237.26 & $A_{1 g}$ & 189.25 \\
\hline & 9.24 & 308.24 & $\mathrm{E}_{\mathrm{g}}$ & 67.35 & 7.86 & 262.13 & $\mathrm{~B}_{1 \mathrm{~g}}$ & 6.55 \\
\hline & 9.50 & 317.00 & $B_{2 g}$ & 3.60 & 9.89 & 329.80 & $\mathrm{~B}_{2 \mathrm{~g}}$ & 39.12 \\
\hline & 11.15 & 372.05 & $\mathrm{E}_{\mathrm{g}}$ & 14.74 & 9.91 & 330.48 & $\mathrm{E}_{\mathrm{g}}$ & 164.92 \\
\hline & 11.46 & 382.32 & $\mathrm{~A}_{1 \mathrm{~g}}$ & 95.52 & 12.29 & 409.81 & $\mathrm{~A}_{1 \mathrm{~g}}$ & 190.55 \\
\hline & 13.36 & 445.72 & $\mathrm{E}_{\mathrm{g}}$ & 43.01 & 12.37 & 412.57 & $\mathrm{E}_{\mathrm{g}}$ & 42.75 \\
\hline & 13.40 & 447.14 & $\mathrm{~B}_{1 \mathrm{~g}}$ & 9.57 & 14.36 & 478.95 & $E_{g}$ & 57.83 \\
\hline & 13.88 & 463.14 & $B_{2 g}$ & 0.09 & 14.84 & 495.17 & $\mathrm{~B}_{1 \mathrm{~g}}$ & 26.13 \\
\hline & 13.92 & 464.27 & $\mathrm{E}_{\mathrm{g}}$ & 0.43 & 15.03 & 501.27 & $\mathrm{~B}_{2 \mathrm{~g}}$ & 0.35 \\
\hline & 15.38 & 513.13 & $\mathrm{~B}_{2 \mathrm{~g}}$ & 2.77 & 15.19 & 506.56 & $\mathrm{E}_{\mathrm{g}}$ & 9.38 \\
\hline & 15.39 & 513.48 & $A_{1 g}$ & 651.68 & 16.58 & 553.19 & $\mathrm{~B}_{1 \mathrm{~g}}$ & 43.09 \\
\hline & 15.65 & 522.04 & $B_{1 g}$ & 60.42 & 17.22 & 574.51 & $A_{1 g}$ & 371.62 \\
\hline & & & & & 17.24 & 575.24 & $\mathrm{~B}_{2 \mathrm{~g}}$ & 0.19 \\
\hline \multirow{16}{*}{$\begin{array}{c}\text { Longitudinal } \\
\text { Optical } \\
\text { Modes }\end{array}$} & 1.64 & 54.66 & $\overline{E_{u}}$ & 0.00 & 1.75 & 58.45 & $\mathrm{~A}_{2 \mathrm{u}}$ & 0.00 \\
\hline & 1.89 & 62.90 & $\mathrm{E}_{\mathrm{u}}$ & 0.00 & 2.02 & 67.42 & $\mathrm{E}_{\mathrm{u}}$ & 0.00 \\
\hline & 2.08 & 69.53 & $\mathrm{~A}_{2 \mathrm{u}}$ & 0.00 & 2.28 & 76.09 & $\mathrm{E}_{\mathrm{u}}$ & 0.00 \\
\hline & 2.43 & 81.22 & $\mathrm{E}_{\mathrm{u}}$ & 0.00 & 2.66 & 88.81 & $\mathrm{E}_{\mathrm{u}}$ & 0.00 \\
\hline & 3.58 & 119.49 & $\mathrm{~A}_{2 \mathrm{u}}$ & 0.00 & 3.48 & 116.21 & $\mathrm{~A}_{2 \mathrm{u}}$ & 0.00 \\
\hline & 3.30 & 109.97 & $E_{u}$ & 0.00 & 4.38 & 146.25 & $\mathrm{E}_{\mathrm{u}}$ & 0.00 \\
\hline & 4.62 & 154.15 & $\mathrm{E}_{\mathrm{u}}$ & 0.00 & 4.97 & 165.82 & $\mathrm{E}_{\mathrm{u}}$ & 0.00 \\
\hline & 5.68 & 189.30 & $\mathrm{E}_{\mathrm{u}}$ & 0.00 & 6.51 & 217.27 & $\mathrm{~A}_{2 \mathrm{u}}$ & 0.00 \\
\hline & 8.46 & 282.21 & $E_{u}$ & 0.00 & 9.01 & 300.45 & $\mathrm{E}_{\mathrm{u}}$ & 0.00 \\
\hline & 10.02 & 334.23 & $\mathrm{E}_{\mathrm{u}}$ & 0.00 & 10.64 & 354.91 & $\mathrm{E}_{\mathrm{u}}$ & 0.00 \\
\hline & 11.95 & 398.66 & $\mathrm{~A}_{2 \mathrm{u}}$ & 0.00 & 12.03 & 401.25 & $\mathrm{E}_{\mathrm{u}}$ & 0.00 \\
\hline & 11.30 & 376.85 & $\mathrm{E}_{\mathrm{u}}$ & 0.00 & 13.17 & 439.26 & $\mathrm{E}_{\mathrm{u}}$ & 0.00 \\
\hline & 13.48 & 449.63 & $\mathrm{E}_{\mathrm{u}}$ & 0.00 & 14.79 & 493.20 & $\mathrm{~A}_{2 \mathrm{u}}$ & 0.00 \\
\hline & 15.05 & 501.93 & $\mathrm{E}_{\mathrm{u}}$ & 0.00 & 16.30 & 543.78 & $\mathrm{~A}_{2 \mathrm{u}}$ & 0.00 \\
\hline & 15.94 & 531.56 & $\mathrm{E}_{\mathrm{u}}$ & 0.00 & 17.41 & 580.79 & $\mathrm{E}_{\mathrm{u}}$ & 0.00 \\
\hline & 16.01 & 534.01 & $\mathrm{~A}_{2 \mathrm{u}}$ & 0.00 & 17.45 & 582.20 & $E_{u}$ & 0.00 \\
\hline
\end{tabular}



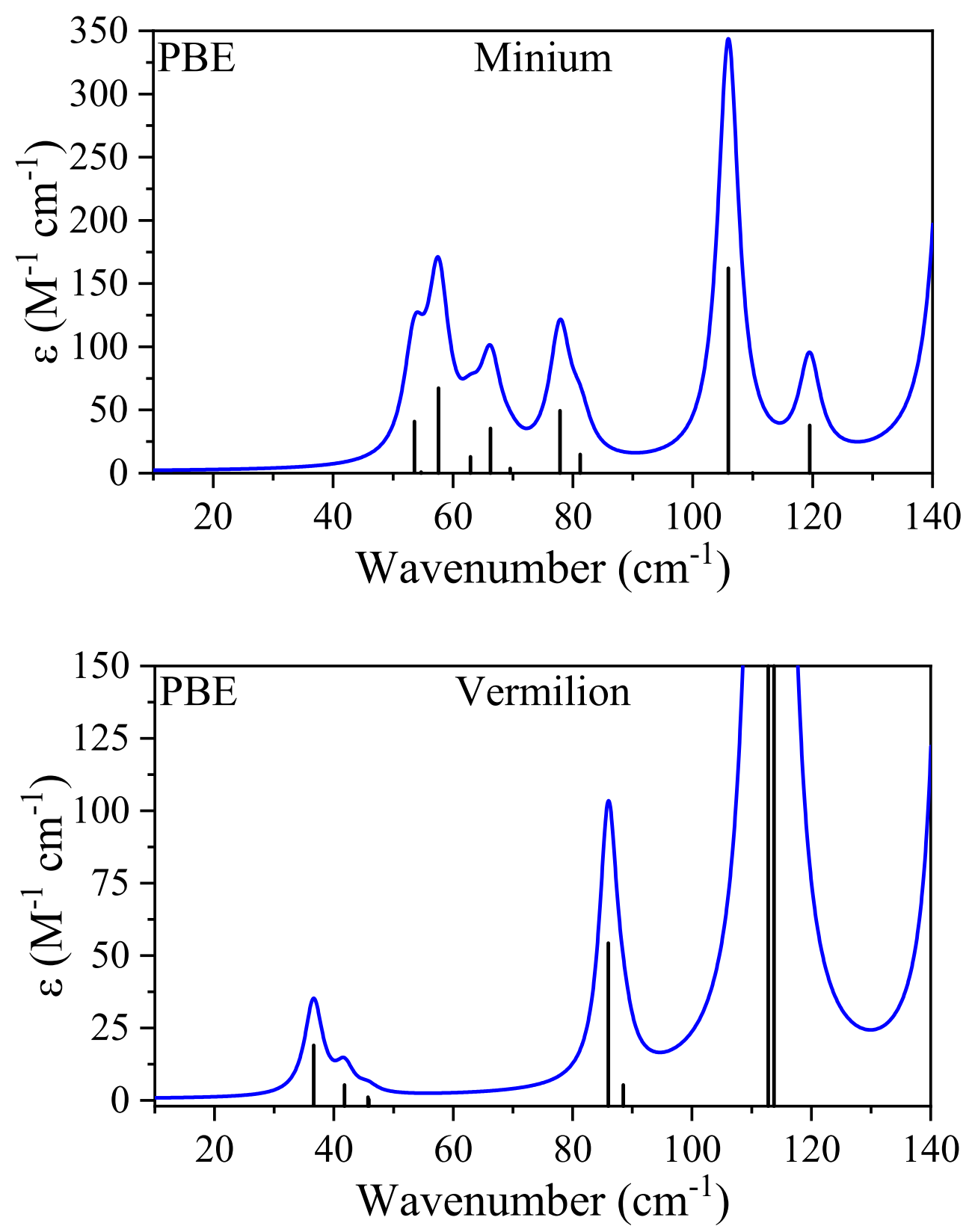

Figure S2. THz-TDS spectra simulated with PBE were convolved using empirically determined Lorentzian line shapes with a FWHM of $2.15 \mathrm{~cm}^{-1}$ for minium (top-panel) and $1.85 \mathrm{~cm}^{-1}$ for vermilion (bottom panel). 
Table S6. Solid-state DFT simulated IR-active frequencies (including LO/TO splitting) for vermilion.

\begin{tabular}{|c|c|c|c|c|c|c|c|c|}
\hline & \multicolumn{4}{|c|}{ PBE } & \multicolumn{4}{|c|}{ PBE0 } \\
\hline & \multicolumn{2}{|c|}{$\begin{array}{c}\text { Frequency } \\
(\mathrm{THz}) \quad\left(\mathrm{cm}^{-1}\right)\end{array}$} & $\begin{array}{c}\text { Mode } \\
\text { Symmetry } \\
\end{array}$ & $\begin{array}{l}\text { Intensity } \\
(\mathrm{km} / \mathrm{mol})\end{array}$ & \multicolumn{2}{|c|}{ Frequency } & $\begin{array}{c}\text { Mode } \\
\text { Symmetry }\end{array}$ & $\begin{array}{l}\text { Intensity } \\
(\mathrm{km} / \mathrm{mol})\end{array}$ \\
\hline \multirow{8}{*}{$\begin{array}{l}\text { Transverse } \\
\text { Optical } \\
\text { Modes }\end{array}$} & 1.10 & 36.61 & $\overline{\mathrm{A}_{2}}$ & 22.09 & 1.05 & 35.08 & $\overline{A_{2}}$ & 32.50 \\
\hline & 1.37 & 45.71 & $\mathrm{E}$ & 1.32 & 1.39 & 46.52 & $\mathrm{E}$ & 3.47 \\
\hline & 2.58 & 85.99 & E & 63.1 & 2.72 & 90.61 & E & 83.67 \\
\hline & 3.38 & 112.78 & $\mathrm{E}$ & 452.67 & 3.34 & 111.27 & E & 401.53 \\
\hline & 3.41 & 113.74 & $\mathrm{~A}_{2}$ & 242.42 & 3.34 & 111.54 & $\mathrm{~A}_{2}$ & 200.40 \\
\hline & 7.85 & 261.98 & $\mathrm{E}$ & 192.11 & 8.61 & 287.04 & $\mathrm{E}$ & 228.19 \\
\hline & 9.44 & 315.02 & $\mathrm{~A}_{2}$ & 365.85 & 10.28 & 342.74 & $\mathrm{~A}_{2}$ & 375.70 \\
\hline & 9.51 & 317.17 & $\mathrm{E}$ & 197.01 & 10.49 & 349.95 & $\mathrm{E}$ & 171.36 \\
\hline \multirow{8}{*}{$\begin{array}{c}\text { Longitudinal } \\
\text { Optical } \\
\text { Modes }\end{array}$} & 1.25 & 41.78 & $\mathrm{~A}_{2}$ & 6.20 & 1.33 & 44.39 & $\mathrm{~A}_{2}$ & 7.60 \\
\hline & 1.37 & 45.84 & E & 0.30 & 1.41 & 46.92 & $\mathrm{E}$ & 0.50 \\
\hline & 2.65 & 88.50 & E & 6.20 & 2.81 & 93.62 & E & 4.20 \\
\hline & 4.45 & 148.46 & $\mathrm{E}$ & 381.00 & 4.60 & 153.53 & $\mathrm{E}$ & 352.50 \\
\hline & 4.28 & 142.73 & $\mathrm{~A}_{2}$ & 188.30 & 4.33 & 144.50 & $\mathrm{~A}_{2}$ & 157.50 \\
\hline & 8.09 & 270.02 & E & 196.70 & 8.94 & 298.28 & E & 233.90 \\
\hline & 9.77 & 325.75 & $\mathrm{E}$ & 322.00 & 10.76 & 358.97 & $\mathrm{E}$ & 297.10 \\
\hline & 10.08 & 336.21 & $\mathrm{~A}_{2}$ & 435.80 & 11.08 & 369.58 & $\mathrm{~A}_{2}$ & 443.60 \\
\hline
\end{tabular}

Table S7. Solid-state DFT simulated Raman-active frequencies and normalized intensities (including LO/TO splitting) for vermilion.

\begin{tabular}{|c|c|c|c|c|c|c|c|c|}
\hline & \multicolumn{4}{|c|}{ PBE } & \multicolumn{4}{|c|}{ PBE0 } \\
\hline & \multicolumn{2}{|c|}{ Frequency } & $\begin{array}{c}\text { Mode } \\
\text { Symmetry }\end{array}$ & $\begin{array}{l}\text { Intensity } \\
(\mathrm{km} / \mathrm{mol})\end{array}$ & $\begin{array}{r}\text { Frec } \\
(\mathrm{THz})\end{array}$ & Frequency & $\begin{array}{c}\text { Mode } \\
\text { Symmetry }\end{array}$ & $\begin{array}{c}\text { Intensity } \\
(\mathrm{km} / \mathrm{mol})\end{array}$ \\
\hline & 1.32 & 44.06 & $\mathrm{~A}_{1}$ & 558.08 & 1.37 & 45.86 & $\mathrm{~A}_{1}$ & 548.63 \\
\hline & 1.37 & 45.71 & $\mathrm{E}$ & 1.93 & 1.39 & 46.52 & $\mathrm{E}$ & 20.81 \\
\hline Transverse & 2.58 & 85.99 & E & 1.93 & 2.72 & 90.61 & $\mathrm{E}$ & 18.91 \\
\hline Optical & 3.38 & 112.78 & E & 68.54 & 3.34 & 111.27 & $\mathrm{E}$ & 204.55 \\
\hline \multirow[t]{3}{*}{ Modes } & 7.12 & 237.46 & $\mathrm{~A}_{1}$ & 1000.00 & 7.86 & 262.05 & $\mathrm{~A}_{1}$ & 1000.00 \\
\hline & 7.85 & 261.98 & E & 115.24 & 8.61 & 287.04 & E & 87.45 \\
\hline & 9.51 & 317.17 & E & 265.78 & 10.49 & 349.95 & E & 293.38 \\
\hline \multirow{8}{*}{$\begin{array}{c}\text { Longitudinal } \\
\text { Optical } \\
\text { Modes }\end{array}$} & 1.25 & 41.78 & $\mathrm{~A}_{2}$ & 0.00 & 1.33 & 44.39 & $\mathrm{~A}_{2}$ & 0.00 \\
\hline & 1.37 & 45.84 & E & 2.10 & 1.41 & 46.92 & E & 20.41 \\
\hline & 2.65 & 88.50 & E & 1.18 & 2.81 & 93.62 & $\mathrm{E}$ & 20.06 \\
\hline & 4.45 & 148.45 & $\mathrm{E}$ & 147.99 & 4.60 & 153.53 & $\mathrm{E}$ & 325.76 \\
\hline & 4.28 & 142.73 & $\mathrm{~A}_{2}$ & 0.00 & 4.33 & 144.50 & $\mathrm{~A}_{2}$ & 0.00 \\
\hline & 8.09 & 270.02 & E & 161.01 & 8.94 & 298.28 & E & 135.86 \\
\hline & 9.77 & 325.75 & $\mathrm{E}$ & 243.13 & 10.76 & 358.97 & $\mathrm{E}$ & 269.32 \\
\hline & 10.08 & 336.21 & $\mathrm{~A}_{2}$ & 0.00 & 11.08 & 369.58 & $\mathrm{~A}_{2}$ & 0.00 \\
\hline
\end{tabular}



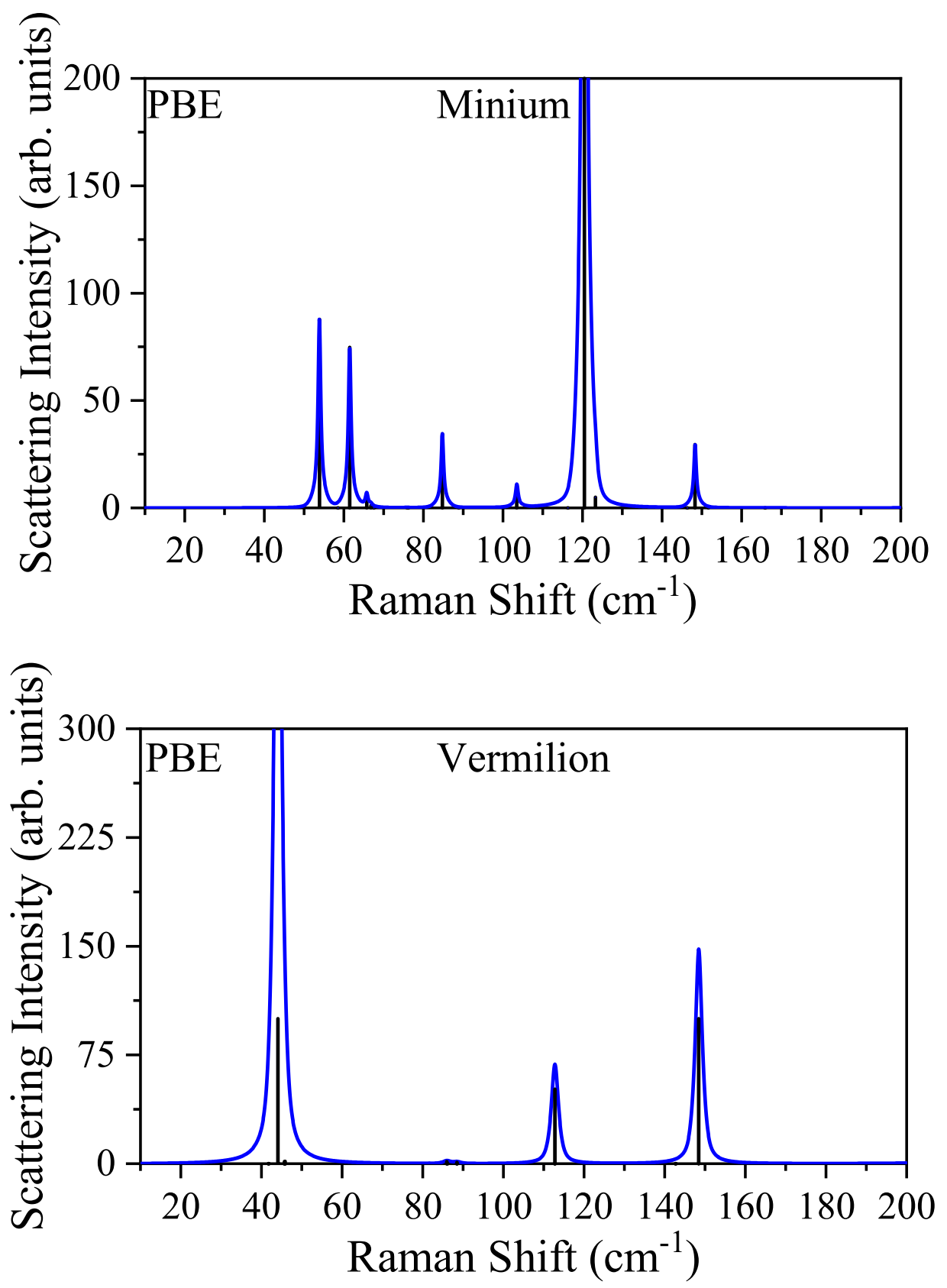

Figure S3. LFRS spectra simulated with PBE were convolved using Voigt profiles for minium (top-panel) and vermilion (bottom panel). 


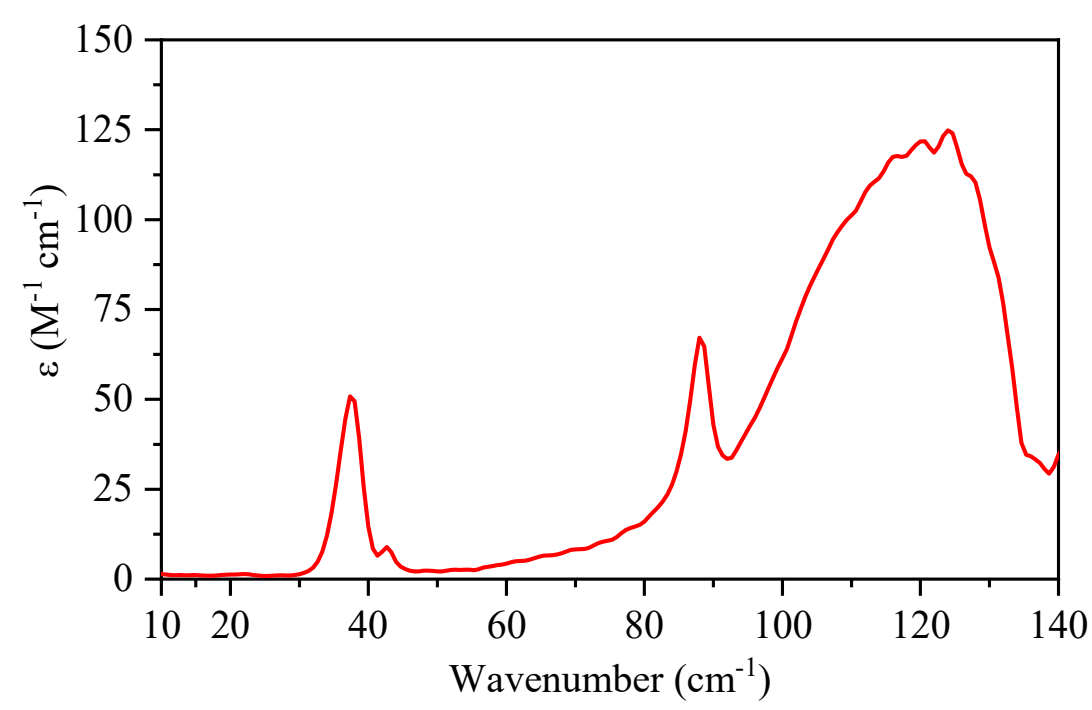

\begin{tabular}{c} 
Vermilion \\
Exp. Peaks $\left(\mathrm{cm}^{-1}\right)$ \\
\hline 37.3 \\
42.7 \\
88.0
\end{tabular}

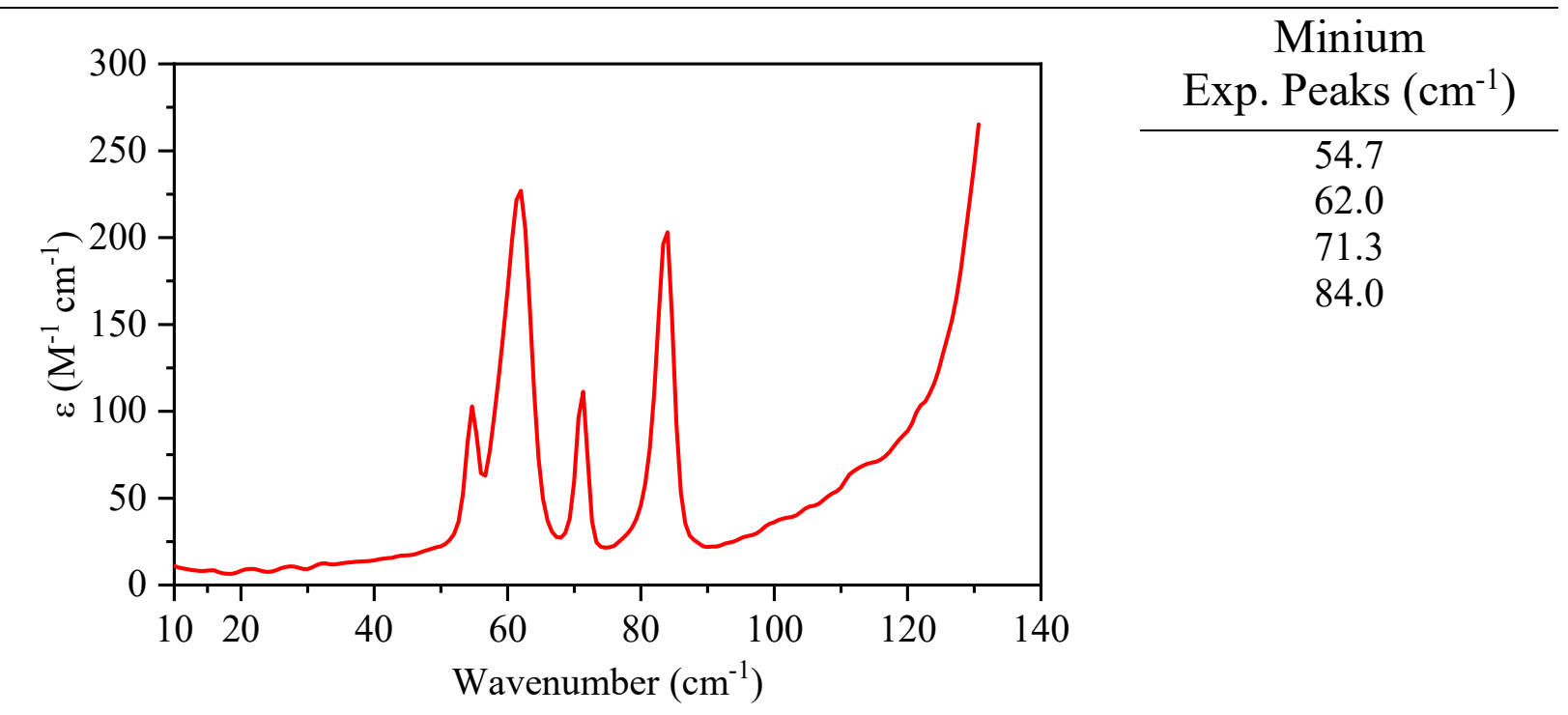

Figure S4. Experimental $290 \mathrm{~K}$ THz-TDS spectra of pure vermilion (top) and minium (bottom). 

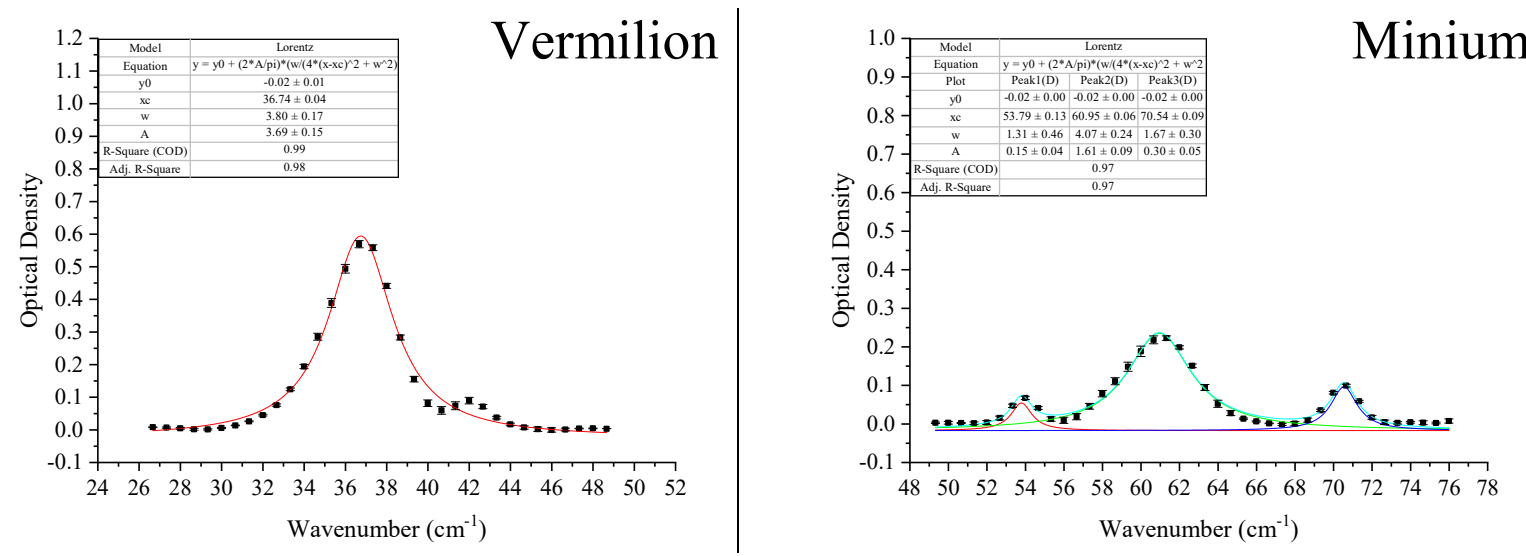

Figure S5. Peak fitting with Lorentzian line shapes of specific THz-TDS spectral range in minium and vermilion mixture sample, $1: 2$, using Origin $2018 \mathrm{~b}^{\mathrm{SR} 3}$.
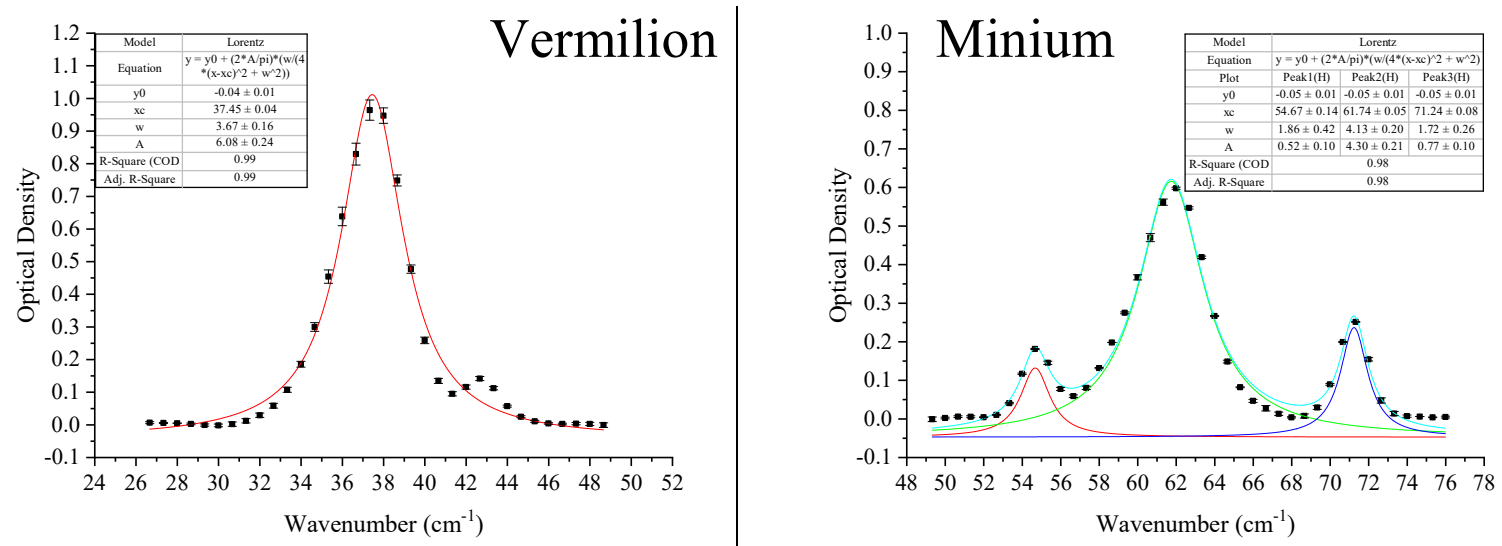

Figure S6. Peak fitting with Lorentzian line shapes of specific THz-TDS spectral range in minium and vermilion mixture sample, $2: 3$, using Origin $2018 \mathrm{~b}^{\mathrm{SR} 3}$.
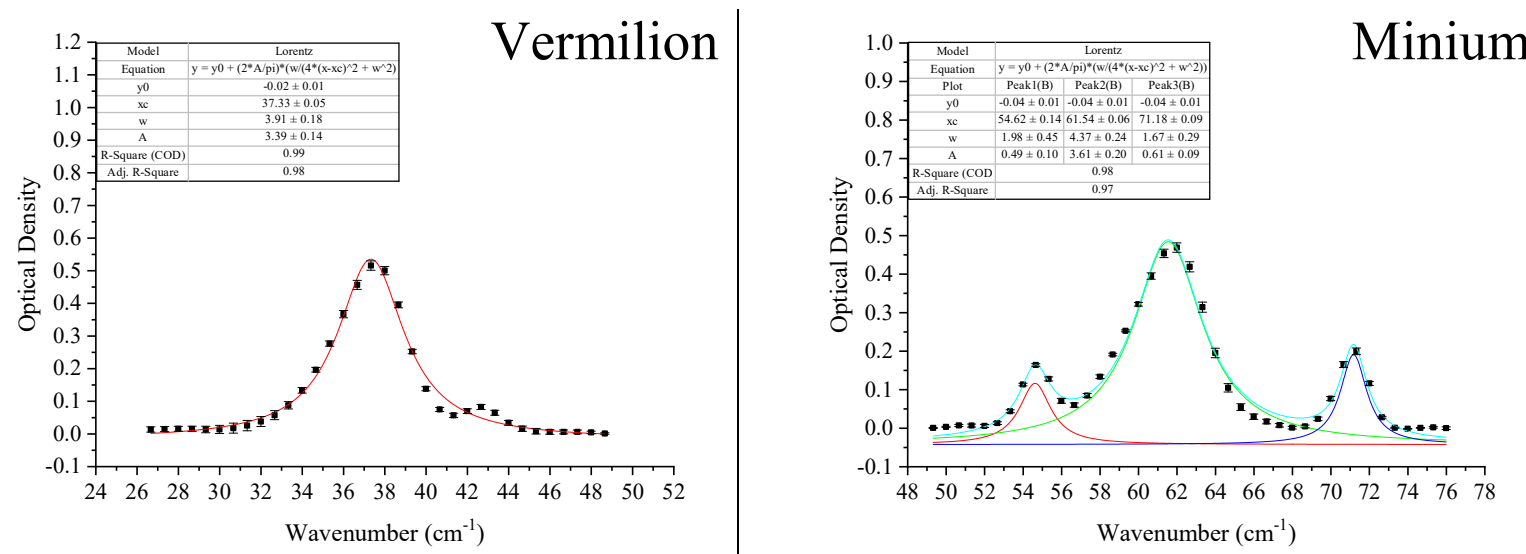

Figure S7. Peak fitting with Lorentzian line shapes of specific THz-TDS spectral range in minium and vermilion mixture sample, $1: 1$, using Origin $2018 b^{\mathrm{SR} 3}$. 

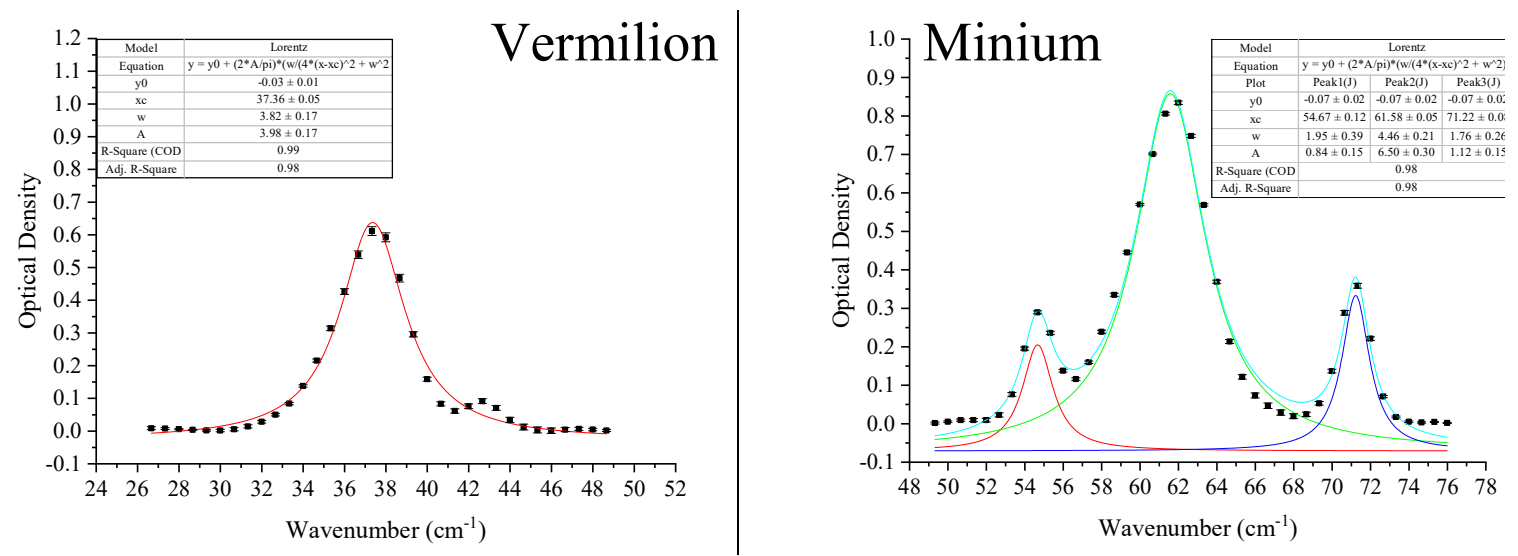

Figure S8. Peak fitting with Lorentzian line shapes of specific THz-TDS spectral range in minium and vermilion mixture sample, $3: 2$, using Origin $2018 \mathrm{~b}^{\mathrm{SR} 3}$.
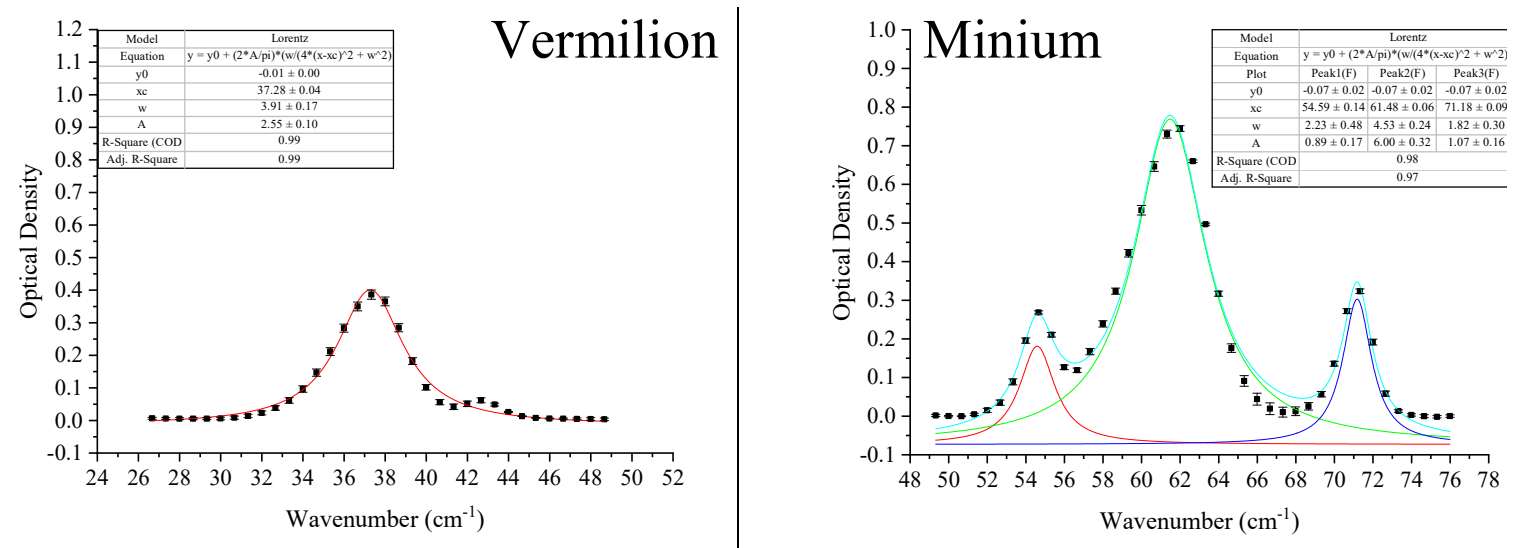

Figure S9. Peak fitting with Lorentzian line shapes of specific THz-TDS spectral range in minium and vermilion mixture sample, $2: 1$, using Origin $2018 \mathrm{~b}^{\mathrm{SR} 3}$. 

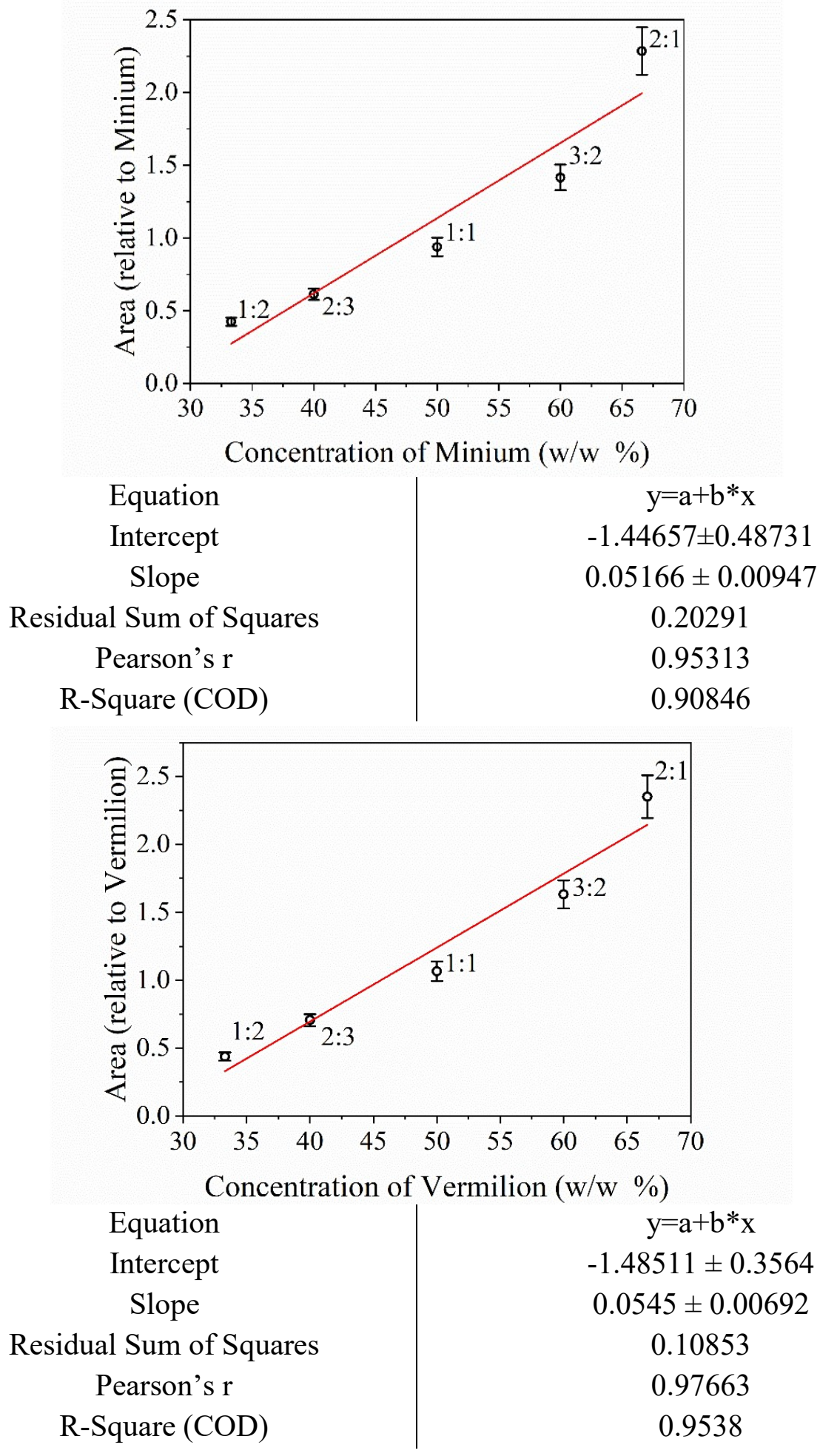

Figure S10. Parameters for linear least squares fit of THz-TDS calibration curves for minium (top) and vermilion (bottom). 


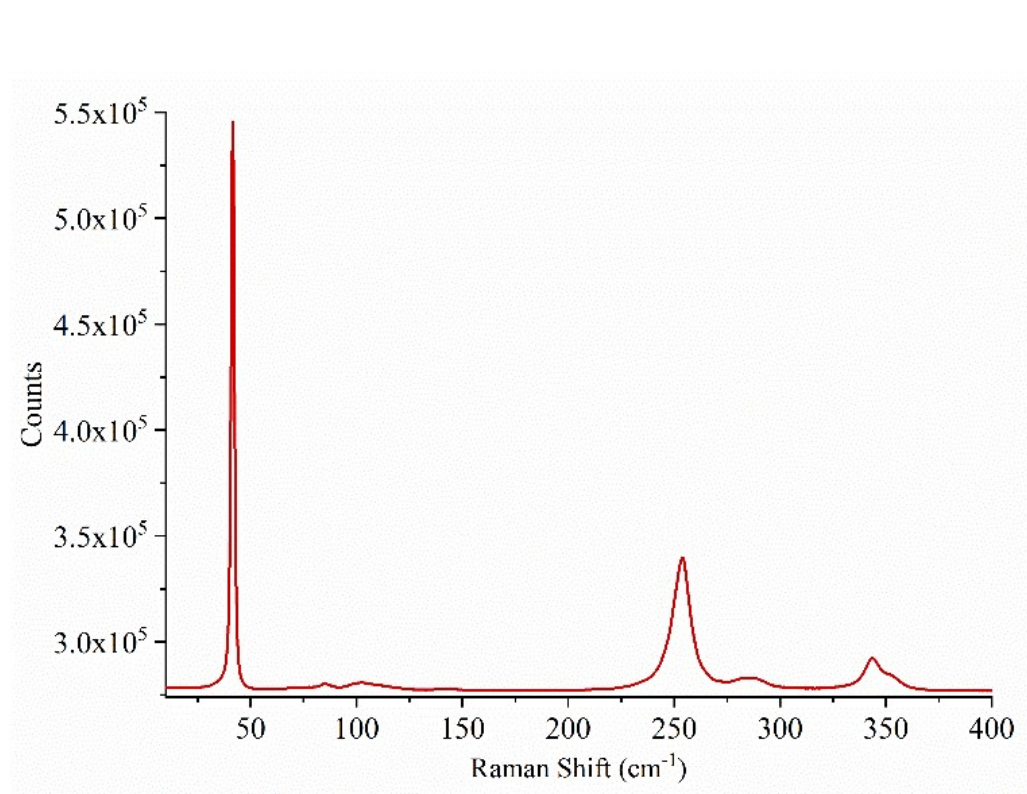

Vermilion

Exp. Peaks $\left(\mathrm{cm}^{-1}\right)$

41.7

84.5

101.9

253.8

282.3

288.4

343.2

351.8

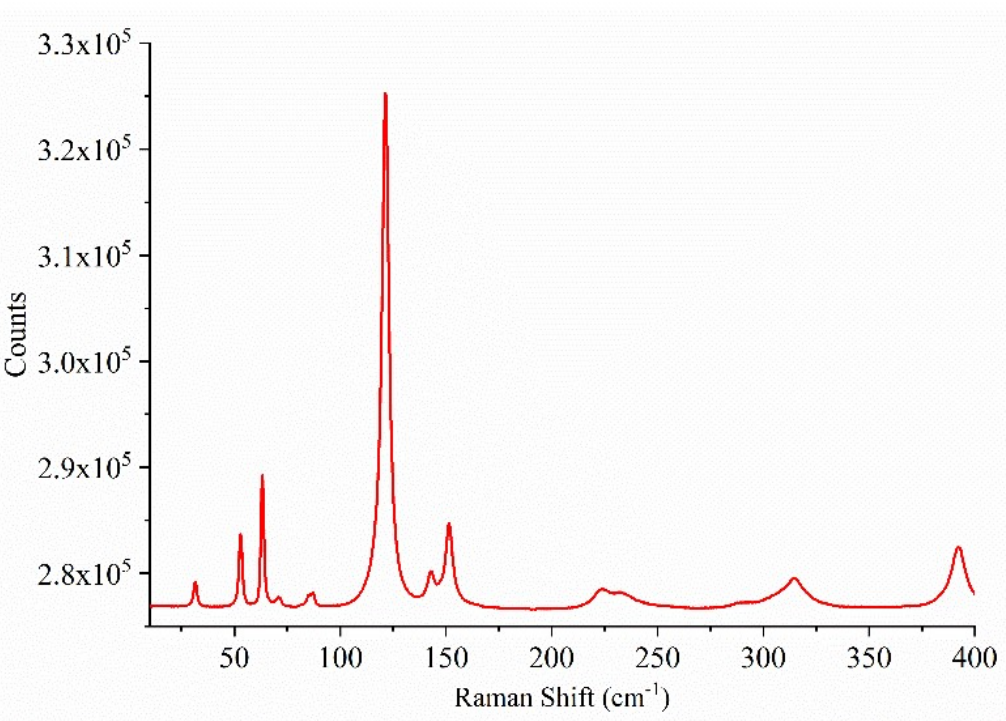

Minium

Exp. Peaks $\left(\mathrm{cm}^{-1}\right)$

31.8

53.4

63.2

71.2

84.9

87.4

121.0

142.7

151.8

223.7

232.8

289.9

314.4

391.8

Figure S11. Experimental 290 K LFRS spectra of pure vermilion (top) and minium (bottom) before normalization. 

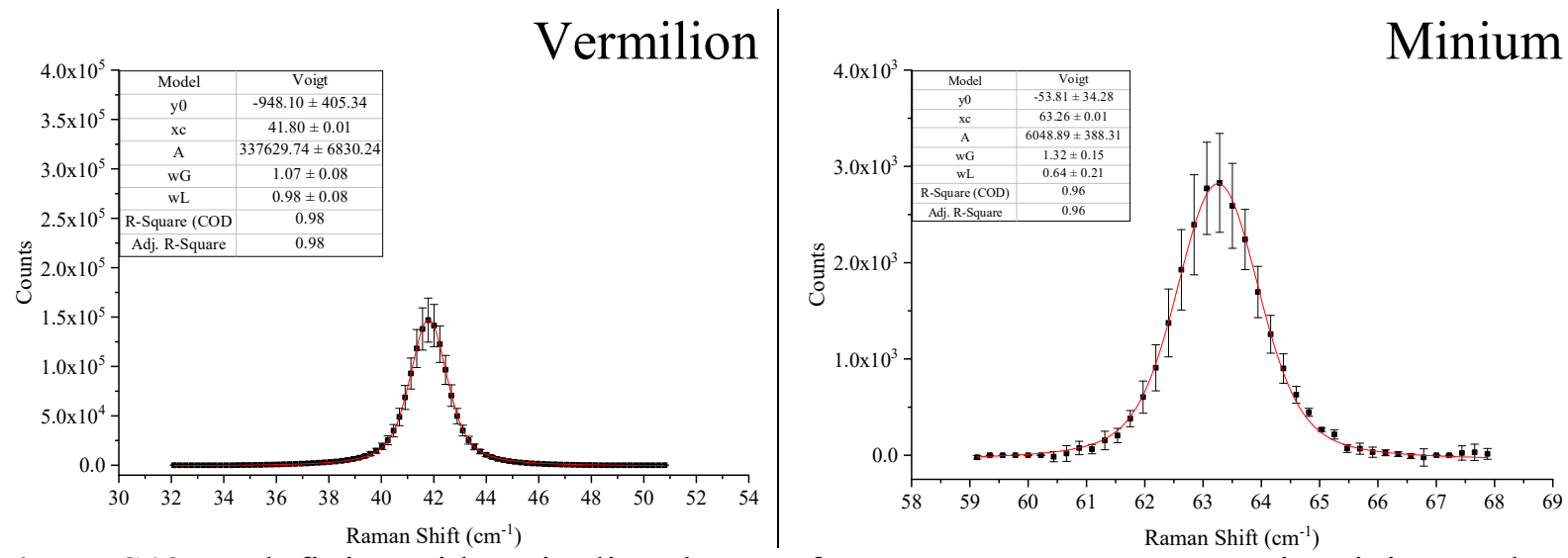

Figure S12. Peak fitting with Voigt line shapes of specific LFRS spectral range in minium and vermilion mixture sample, $1: 2$, using Origin $2018 \mathrm{~b}^{\mathrm{SR} 3}$.
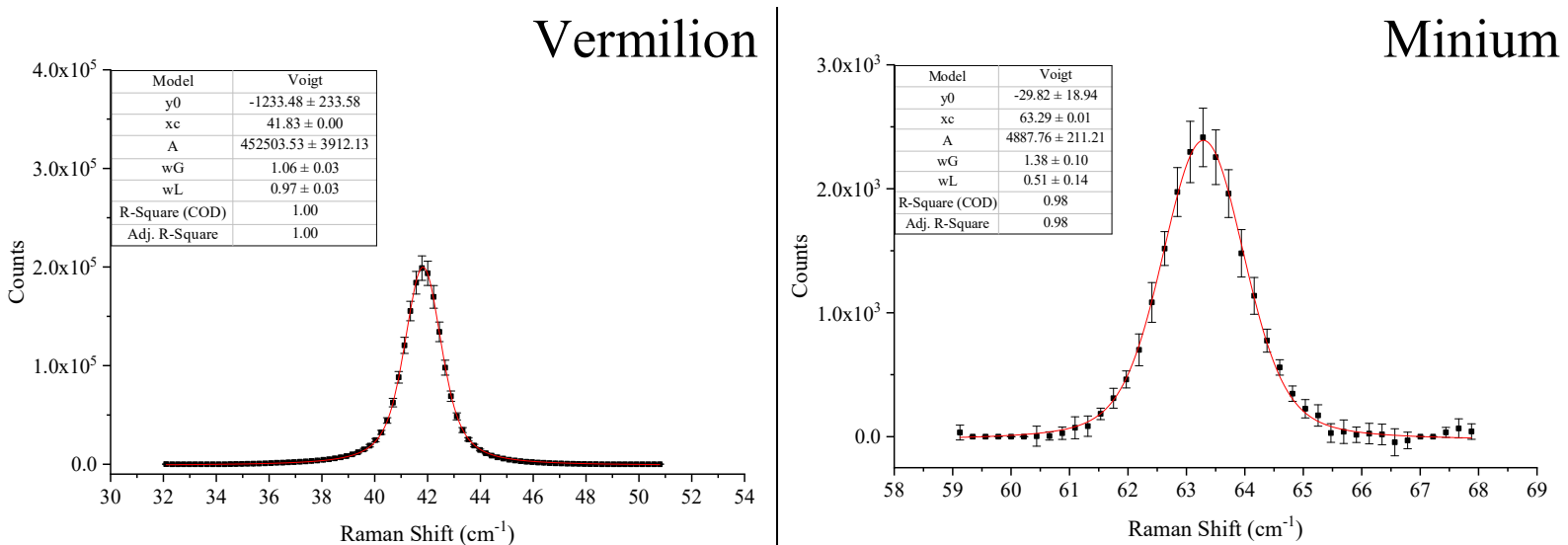

Figure S13. Peak fitting with Voigt line shapes of specific LFRS spectral range in minium and vermilion mixture sample, $2: 3$, using Origin $2018 b^{\text {SR3 }}$.
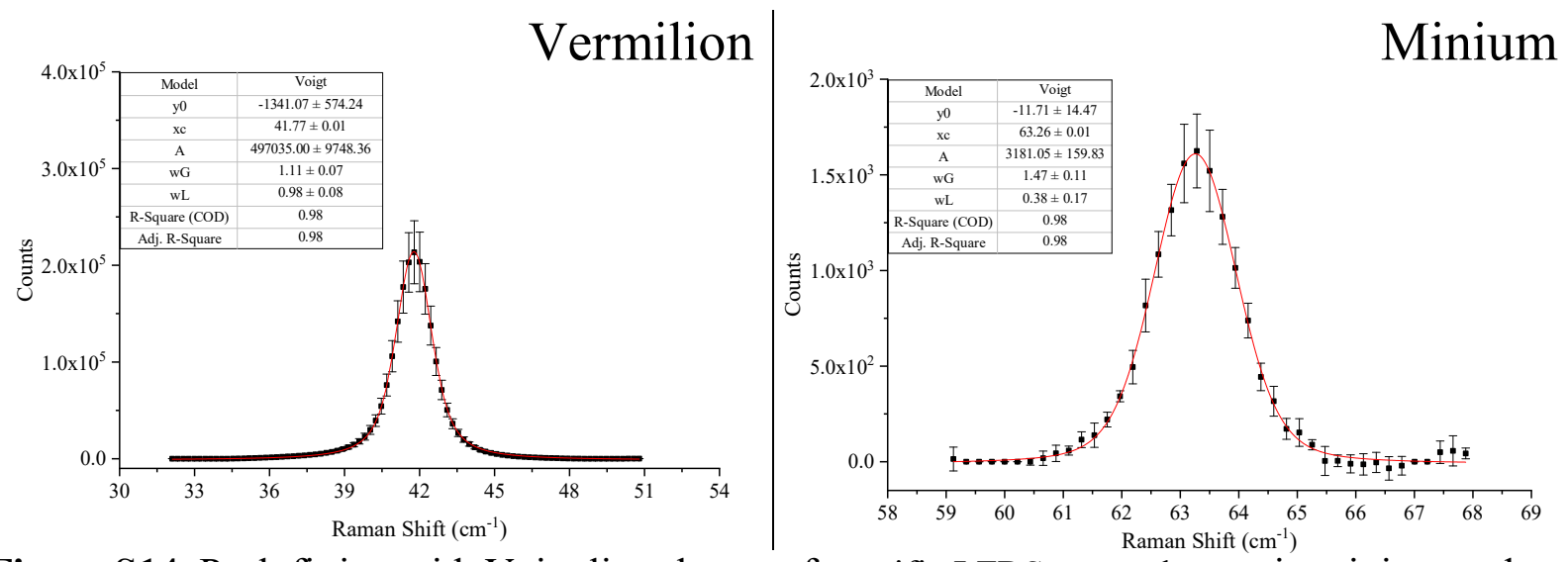

Figure S14. Peak fitting with Voigt line shapes of specific LFRS spectral range in minium and vermilion mixture sample, $1: 1$, using Origin $2018 b^{\text {SR3 }}$. 

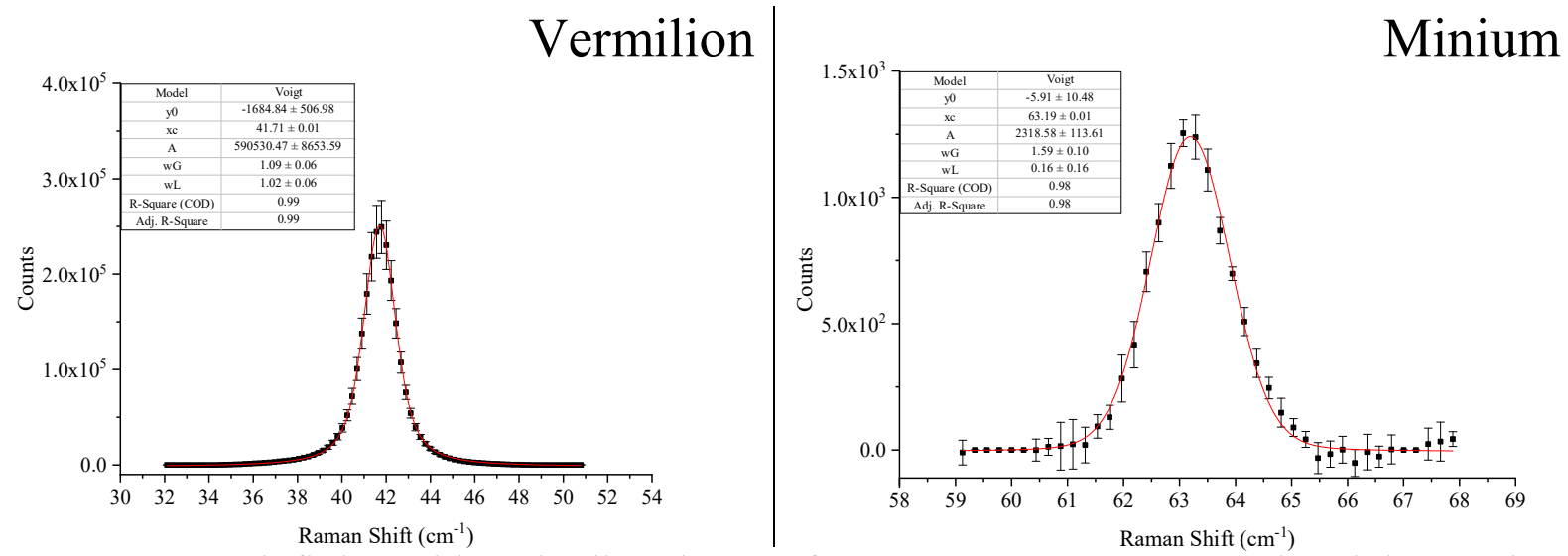

Figure S15. Peak fitting with Voigt line shapes of specific LFRS spectral range in minium and vermilion mixture sample, $3: 2$, using Origin $2018 b^{\mathrm{SR} 3}$.

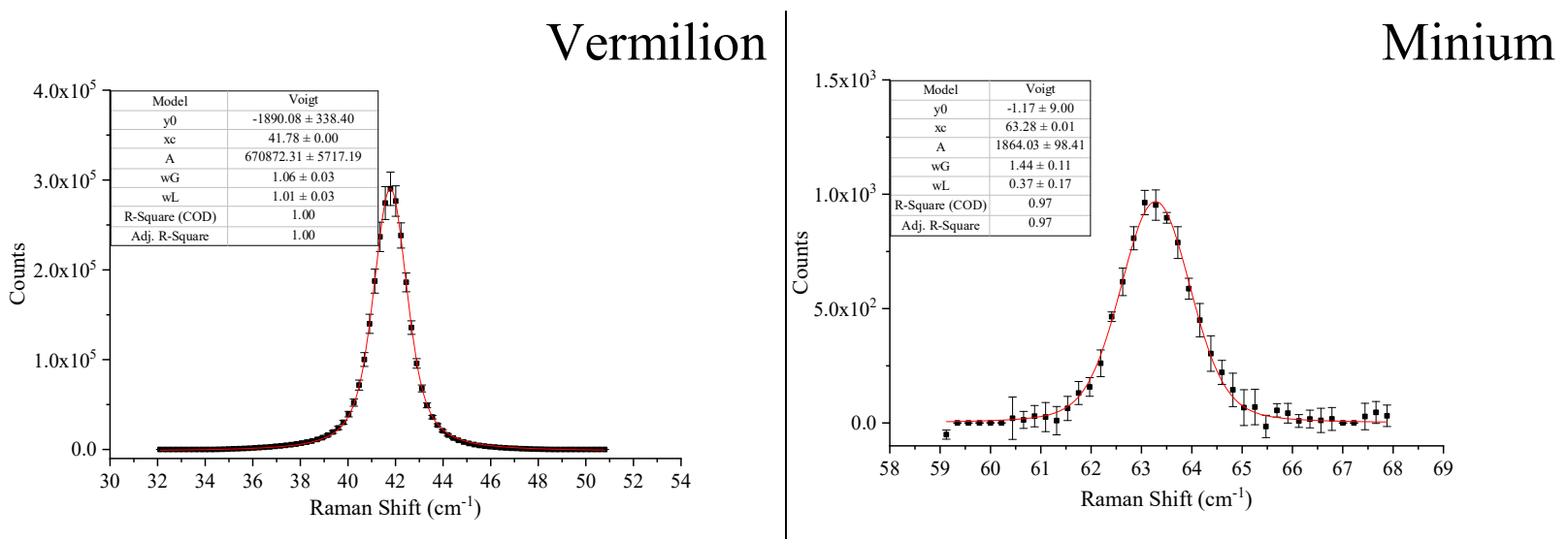

Figure S16. Peak fitting with Voigt line shapes of specific LFRS spectral range in minium and vermilion mixture sample, $2: 1$, using Origin $2018 b^{\mathrm{SR} 3}$. 

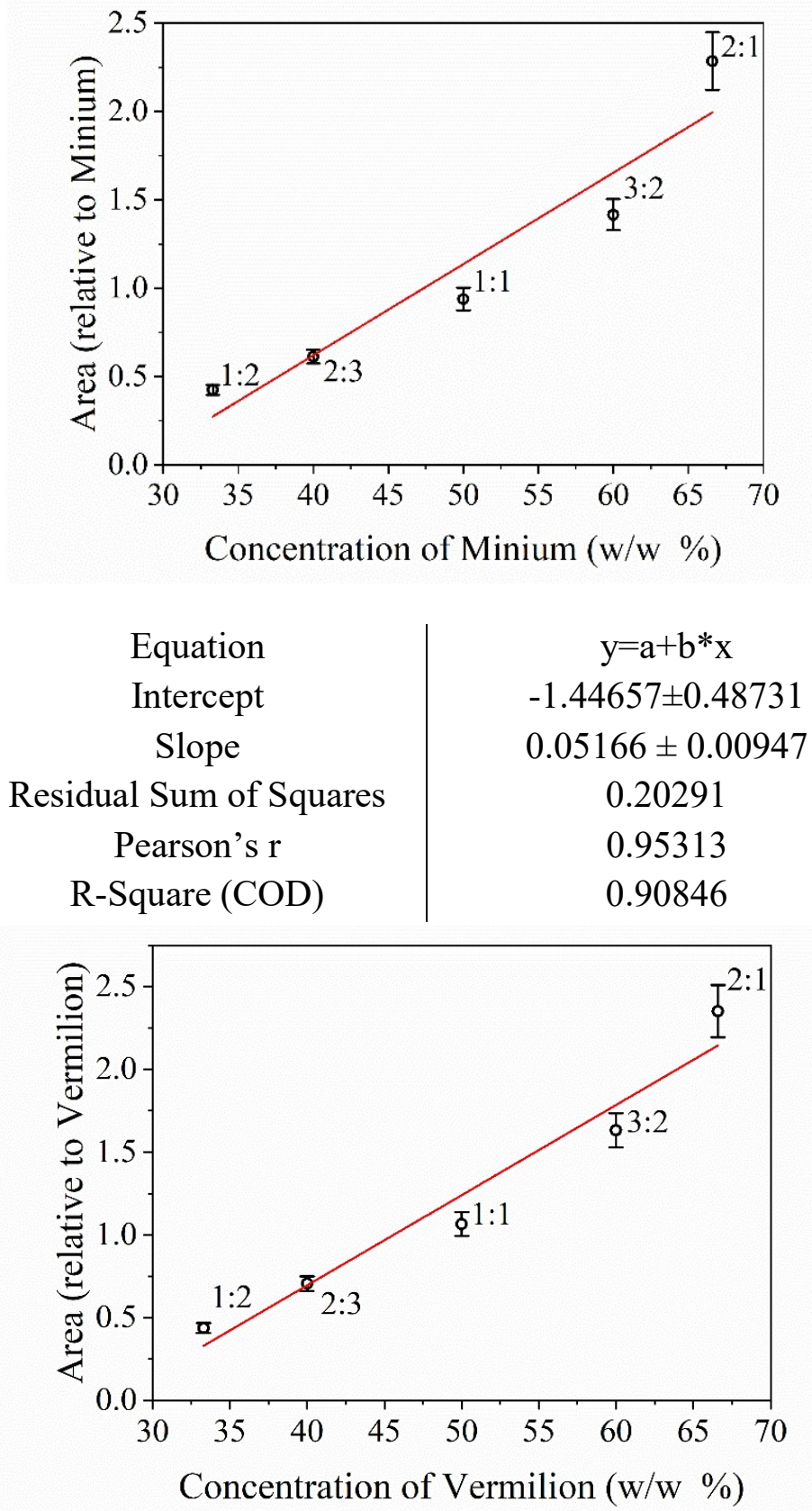

\begin{tabular}{c|c} 
Equation & $\mathrm{y}=\mathrm{a}+\mathrm{b}^{*} \mathrm{x}$ \\
Intercept & $-1.48511 \pm 0.3564$ \\
Slope & $0.0545 \pm 0.00692$ \\
Residual Sum of Squares & 0.10853 \\
Pearson's r & 0.97663 \\
R-Square (COD) & 0.9538
\end{tabular}

Figure S17. Parameters for linear least squares fit of LFRS calibration curves for minium (top) and vermilion (bottom). 
Table S8. Experimental ${ }^{\mathrm{SR} 2}$ and solid-state DFT calculated bonds lengths and angles for minium asymmetric unit.

\begin{tabular}{cccccc}
\hline Bond Length $(\AA)$ & \multicolumn{5}{c}{ Exp. } \\
\hline Atom1 & Atom2 & \multicolumn{5}{c}{ PBE } & PBE0 \\
\hline $\mathrm{Pb} 4+$ & $\mathrm{O} 1$ & 2.13 & 2.18 & 2.14 \\
$\mathrm{~Pb} 4+$ & $\mathrm{O} 2$ & 2.21 & 2.26 & 2.20 \\
$\mathrm{~Pb} 2+$ & $\mathrm{O} 2$ & 2.73 & 2.38 & 2.73 \\
$\mathrm{~Pb} 2+$ & $\mathrm{O} 1$ & 2.22 & 2.27 & 2.23 \\
& & $\mathrm{RMSD}$ & 0.18 & 0.01 \\
\hline Bond Angle $\left({ }^{\circ}\right)$ & & & Exp. & Calcd. \\
\hline Atom1 & Atom2 & Atom3 & & PBE & PBE0 \\
\hline $\mathrm{Pb} 4+$ & $\mathrm{O} 1$ & $\mathrm{~Pb} 2+$ & 112.32 & 111.25 & 112.28 \\
$\mathrm{~Pb} 2+$ & $\mathrm{O} 2$ & $\mathrm{~Pb} 4+$ & 93.51 & 94.84 & 94.00 \\
$\mathrm{O} 1$ & $\mathrm{~Pb} 4+$ & $\mathrm{O} 2$ & 83.35 & 82.42 & 83.31 \\
$\mathrm{O} 1$ & $\mathrm{~Pb} 2+$ & $\mathrm{O} 2$ & 70.56 & 71.28 & 70.21 \\
& & & RMSD & 1.04 & 0.30
\end{tabular}

Table S9. Experimental ${ }^{\mathrm{SR} 1}$ and solid-state DFT calculated bonds length for vermilion asymmetric unit.

\begin{tabular}{ccccc}
\hline Bond Length $(\AA)$ & & Exp. & \multicolumn{2}{c}{ Calcd. } \\
\hline Atom 1 & Atom2 & & PBE & PBE0 \\
\hline $\mathrm{S}$ & $\mathrm{Hg}$ & $\begin{array}{c}2.37 \\
\text { \% Error }\end{array}$ & 2.44 & 2.41 \\
& & & 1.73 & \\
& & & &
\end{tabular}




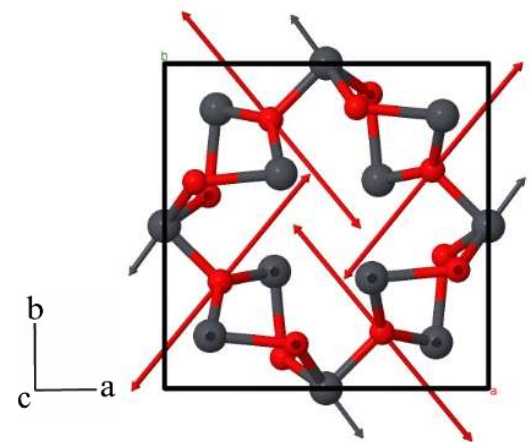

Figure S18. Eigenvector plot for Raman-active mode, $22.43 \mathrm{~cm}^{-1}$, in minium.

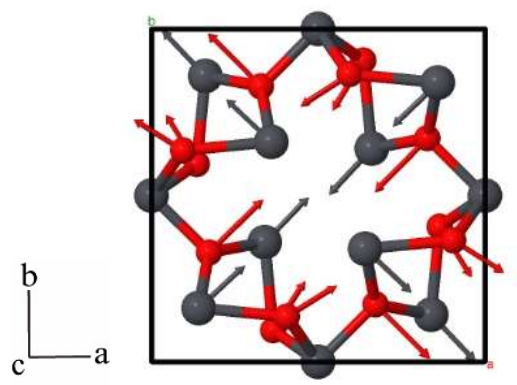

Figure S19. Eigenvector plot for Raman-active mode, $57.37 \mathrm{~cm}^{-1}$, in minium.

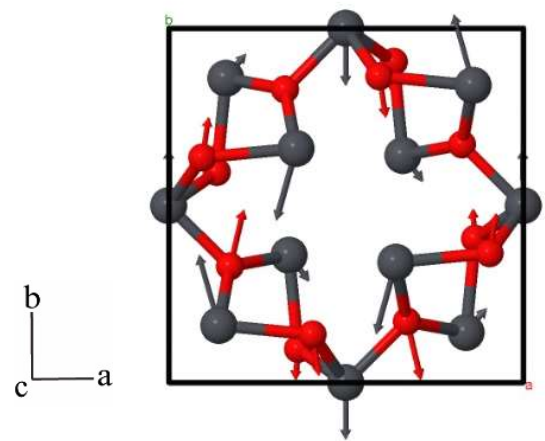

Figure S20. Eigenvector plot for IR-active mode, $58.22 \mathrm{~cm}^{-1}$, in minium.

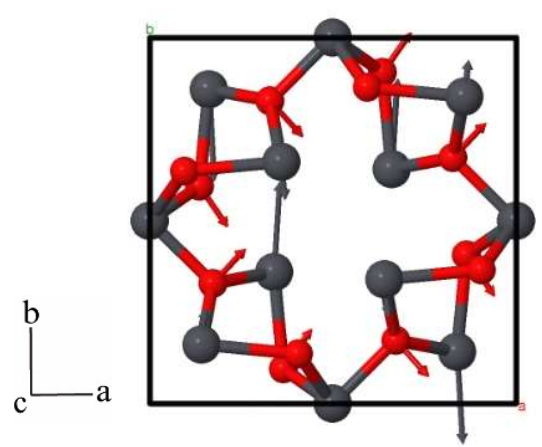

Figure S21. Eigenvector plot for IR-active mode, $60.23 \mathrm{~cm}^{-1}$, in minium. 


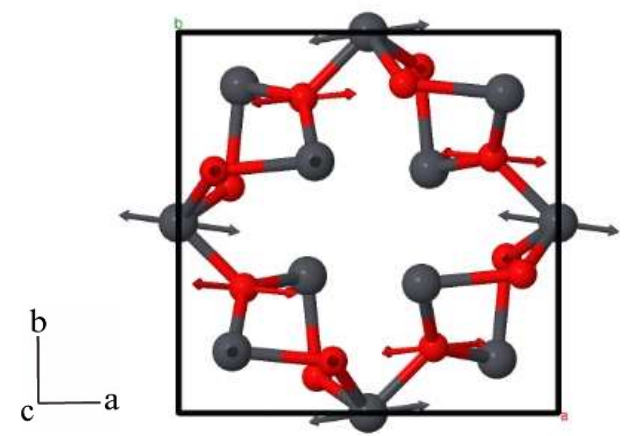

Figure S22. Eigenvector plot for Raman-active mode, $66.95 \mathrm{~cm}^{-1}$, in minium.

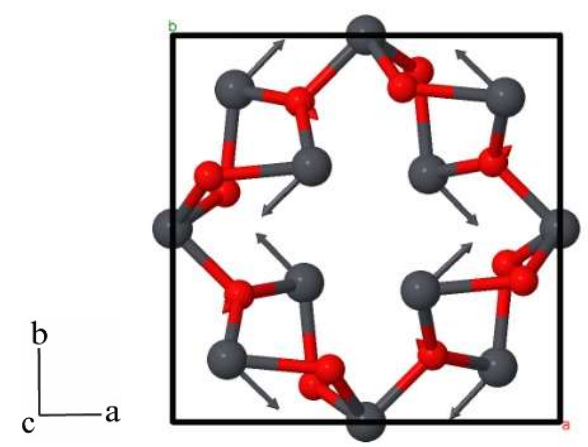

Figure S23. Eigenvector plot for Raman-active mode, $72.11 \mathrm{~cm}^{-1}$, in minium.

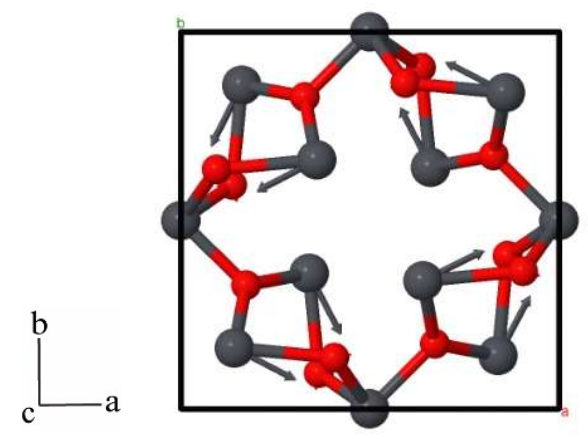

Figure S24. Eigenvector plot for Raman-active mode, $72.91 \mathrm{~cm}^{-1}$, in minium.

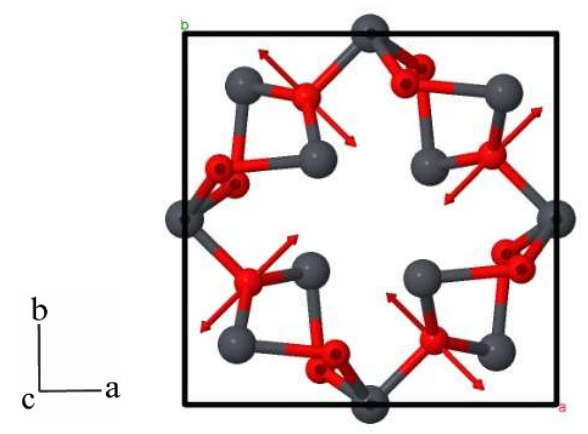

Figure S25. Eigenvector plot for IR-active mode, $74.17 \mathrm{~cm}^{-1}$, in minium. 


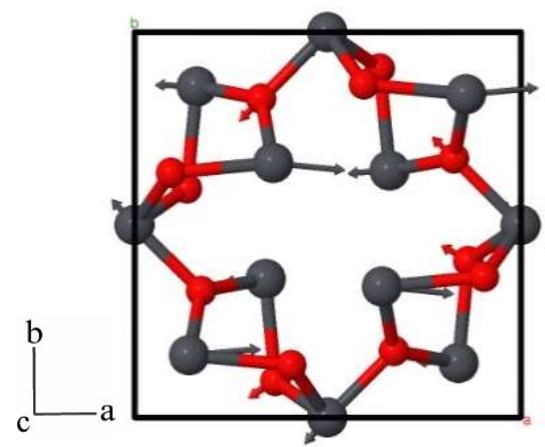

Figure S26. Eigenvector plot for IR-active mode, $85.77 \mathrm{~cm}^{-1}$, in minium.

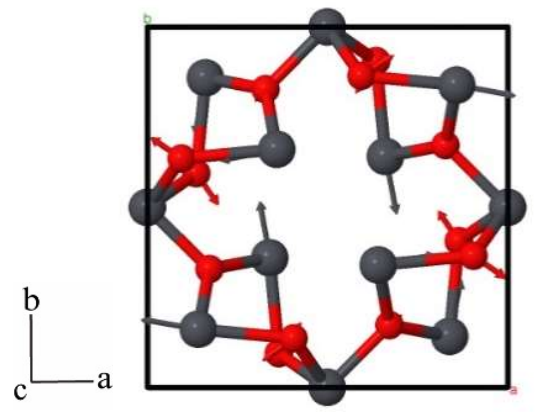

Figure S27. Eigenvector plot for Raman -active mode, $88.06 \mathrm{~cm}^{-1}$, in minium.

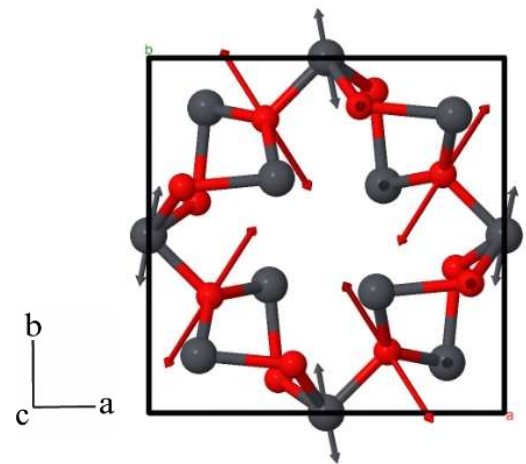

Figure S28. Eigenvector plot for Raman -active mode, $89.24 \mathrm{~cm}^{-1}$, in minium.

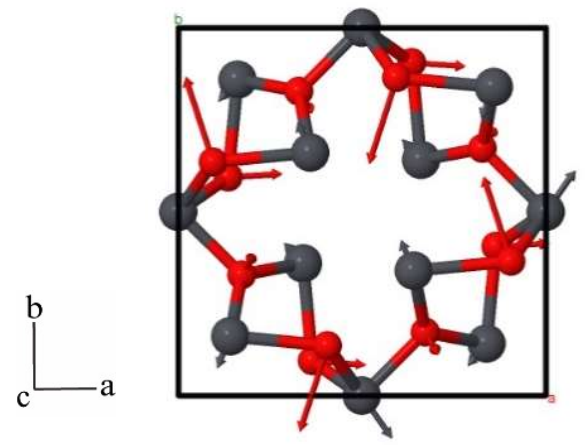

Figure S29. Eigenvector plot for IR-active mode, $116.19 \mathrm{~cm}^{-1}$, in minium. 


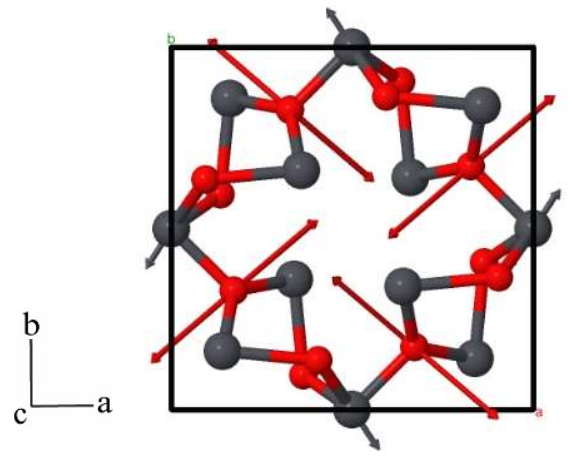

Figure S30. Eigenvector plot for Raman-active mode, $121.67 \mathrm{~cm}^{-1}$, in minium.

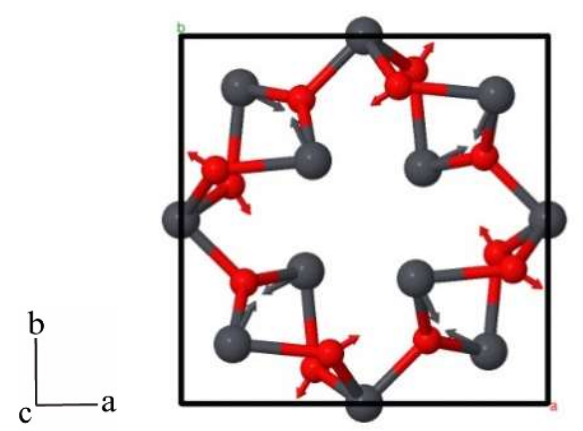

Figure S31. Eigenvector plot for Raman-active mode, $128.60 \mathrm{~cm}^{-1}$, in minium.

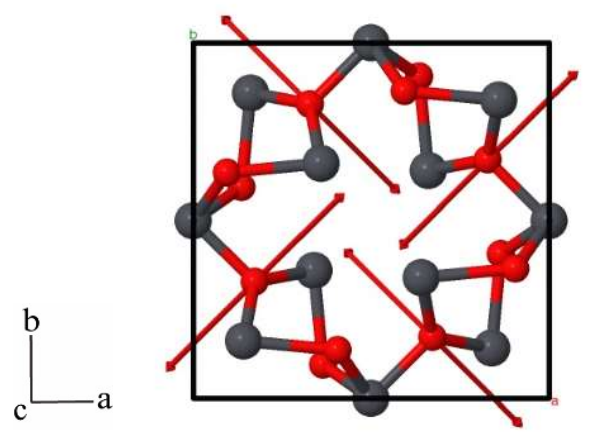

Figure S32. Eigenvector plot for IR-active mode, $130.83 \mathrm{~cm}^{-1}$, in minium.

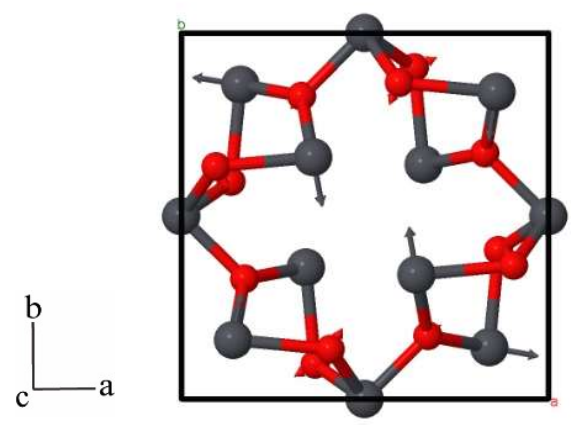

Figure S33. Eigenvector plot for Raman-active mode, $132.15 \mathrm{~cm}^{-1}$, in minium. 


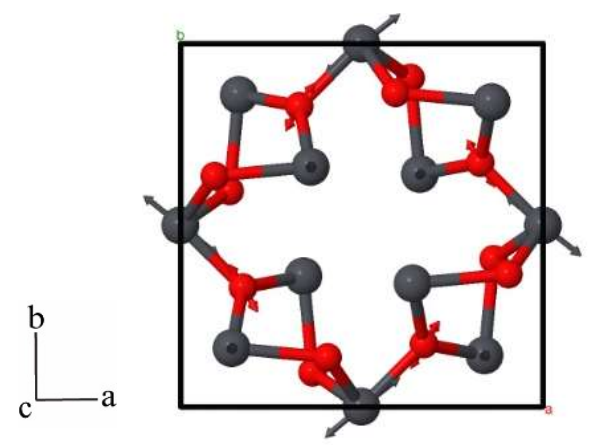

Figure S34. Eigenvector plot for Raman-active mode, $158.79 \mathrm{~cm}^{-1}$, in minium.

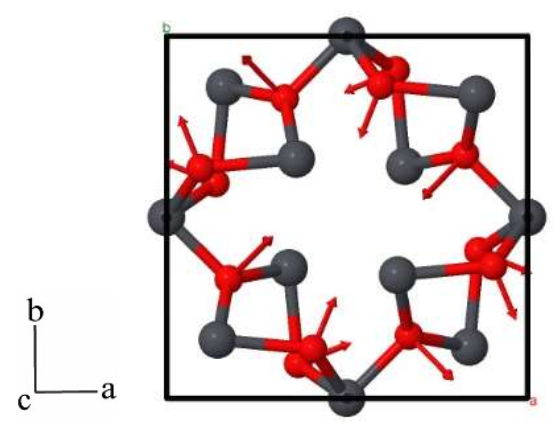

Figure S35. Eigenvector plot for Raman-active mode, $169.84 \mathrm{~cm}^{-1}$, in minium. 


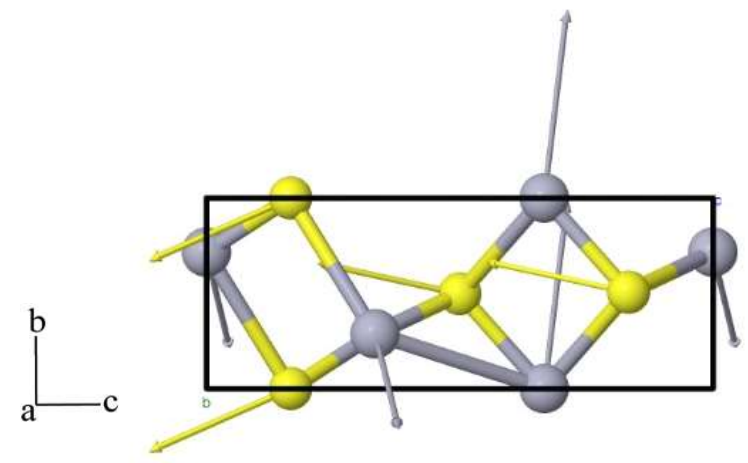

Figure S36. Eigenvector plot for IR-active mode, $35.08 \mathrm{~cm}^{-1}$, in vermilion.

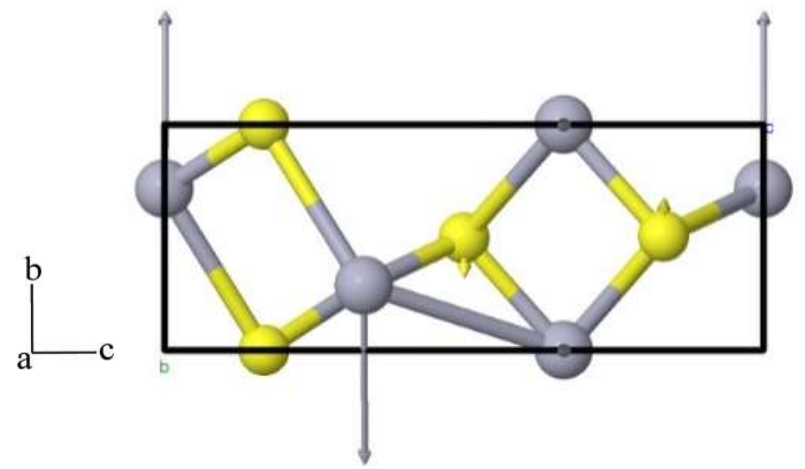

Figure S37. Eigenvector plot for Raman-active mode, $45.86 \mathrm{~cm}^{-1}$, in vermilion.

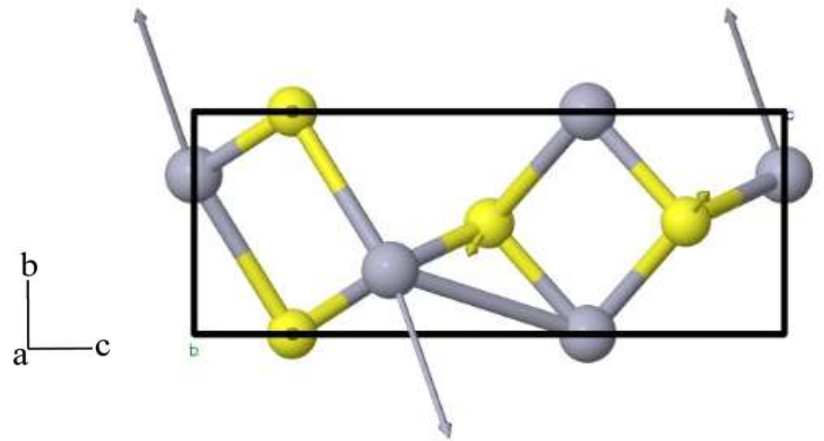

Figure S38. Eigenvector plot for Raman \& IR-active mode, $46.52 \mathrm{~cm}^{-1}$, in vermilion.

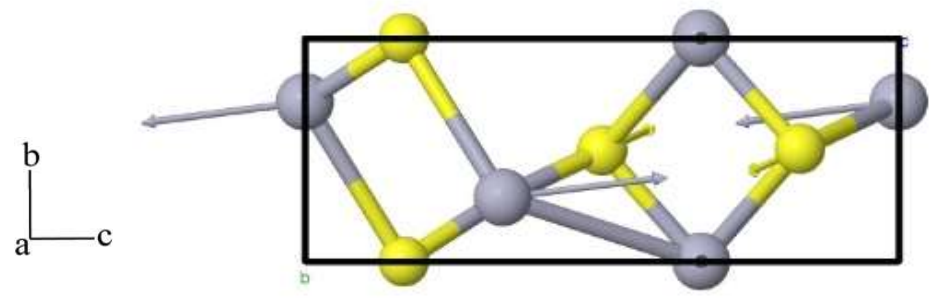

Figure S39. Eigenvector plot for Raman \& IR-active mode, $90.61 \mathrm{~cm}^{-1}$, in vermilion. 


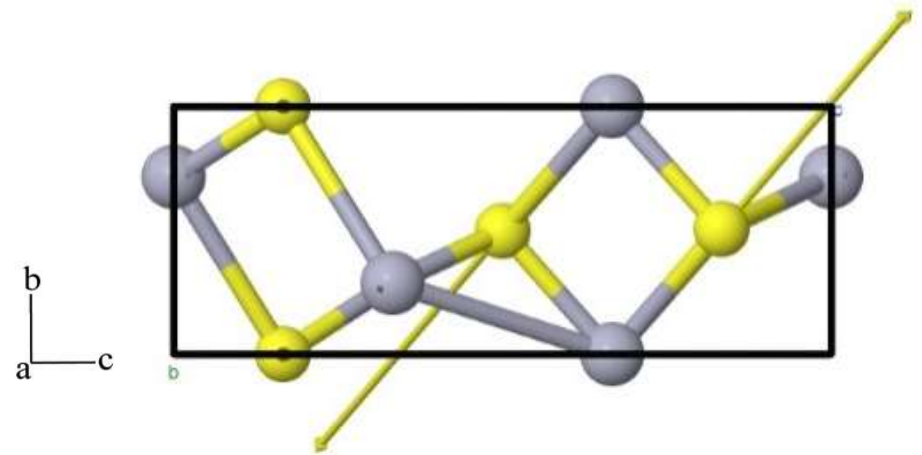

Figure S40. Eigenvector plot for Raman \& IR-active mode, $111.27 \mathrm{~cm}^{-1}$, in vermilion.

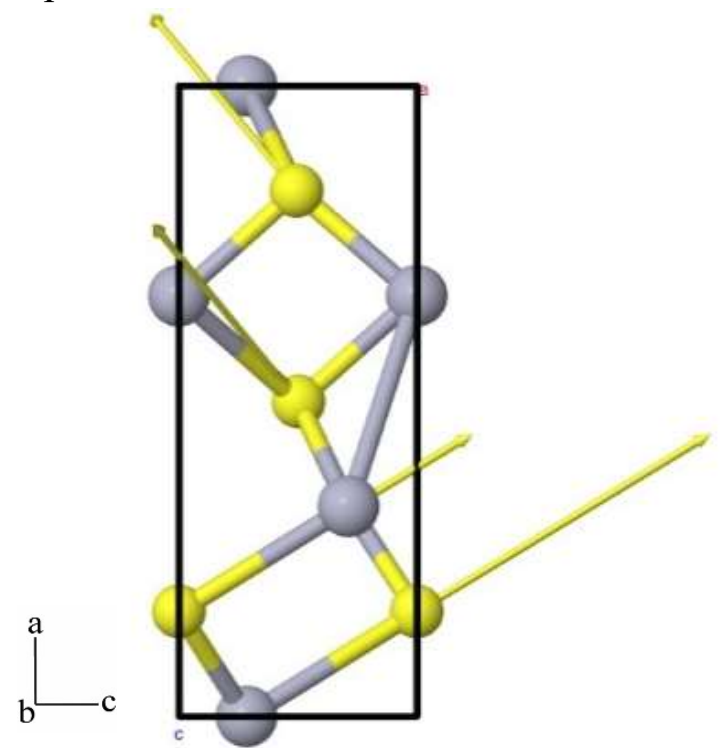

Figure S41. Eigenvector plot for IR-active mode, $111.54 \mathrm{~cm}^{-1}$, in vermilion. 